University of Warwick institutional repository: http://go.warwick.ac.uk/wrap This paper is made available online in accordance with publisher policies. Please scroll down to view the document itself. Please refer to the repository record for this item and our policy information available from the repository home page for further information.

To see the final version of this paper please visit the publisher's website. Access to the published version may require a subscription.

Author(s): MÍRIAM MANOEL and IAN STEWART

Article Title: THE CLASSIFICATION OF BIFURCATIONS WITH HIDDEN SYMMETRIES

Year of publication: 2000 Link to published version: http://dx.doi.org/10.1112/S0024611500012156

Publisher statement: None 


\title{
THE CLASSIFICATION OF BIFURCATIONS WITH HIDDEN SYMMETRIES
}

\author{
MÍRIAM MANOEL and IAN STEWART
}

[Received 15 April 1998; revised 17 November 1998]

\section{Introduction}

Within the last fifteen years it has become apparent that certain kinds of bifurcation problem can be understood by exploiting 'hidden' symmetries related to an extended problem. For example, the one-parameter bifurcation of steady or periodic solutions to certain elliptic partial differential equations with Neumann or Dirichlet boundary conditions can be seen as part of the more general question of solutions with periodic boundary conditions. This point was first realized by Fujii et al. [9]. Many authors have since developed this idea to understand certain bifurcation phenomena observed in the original problem that would not be expected if only the symmetries of the domain were taken into account. The source of non-genericity in these bifurcations is the existence of an extended problem, defined on a larger domain and having a larger symmetry group. Field et al. [8] establish this extension property for partial differential equations

$$
u_{t}+F(u, \lambda)=0
$$

defined by second-order quasilinear elliptic operators in divergent form under Neumann or Dirichlet boundary conditions on a manifold $\mathscr{D}$. They present a large class of pairs of manifolds $\mathscr{D} \subset \widetilde{\mathscr{D}}$ where extra symmetries obtained from the extension to the larger manifold change the genericity of the original problem in the smaller manifold. They let $\widetilde{\mathscr{D}}$ be any smooth, compact, connected, Riemannian $n$-manifold without boundary acted upon by a group of reflections which divides $\widetilde{\mathscr{D}}$ into several connected components. The smaller manifold with boundary, $\mathscr{D}$, is one of these connected components. They also show that the extension procedure preserves the regularity of solutions of (1.0.1), so smooth solutions on $\mathscr{D}$ correspond to smooth solutions on $\widetilde{\mathscr{D}}$ that are invariant under these reflections. In this setting, they prove that symmetries present in $\widetilde{\mathscr{D}}$ change the generic properties of the original problem defined on $\mathscr{D}$, in the sense that unexpected degeneracies in the original problem are explained by symmetries that occur in the extended domain but are not apparent in the original problem.

Crawford et al. [5] consider reaction-diffusion equations invariant under translations and reflections of the domain with Neumann or Dirichlet boundary conditions. For this case there is a natural way to extend the domain to introduce a larger symmetry group, and the authors discuss how these symmetries are related to the unexpected behaviour present in the original problem. Gomes and Stewart [14] apply the extension results of [8] to study the mode interaction of

The first author was partially supported by a grant from $\mathrm{CNPq}$, Brazil.

1991 Mathematics Subject Classification: 58C27, 58F14.

Proc. London Math. Soc. (3) 80 (2000) 198-234. (C) London Mathematical Society 2000 
two steady state modes for (1.0.1), where $u: \mathbb{R}^{N} \times \mathbb{R} \rightarrow \mathbb{R}, \lambda$ is the bifurcation parameter, and $\mathscr{P}$ is an elliptic operator defined on an appropriate function space. The motivation is the study of steady solutions defined on an $N$-dimensional rectangle satisfying Neumann boundary conditions. They prove that for most mode numbers the Liapunov-Schmidt reduced equations for this problem have exactly the same form as the equations deduced by Armbruster and Dangelmayr $[2,7]$ when $N=1$. In $\S 4$ we use the results of [14] to explain how the singularity theory for this problem can similarly be reduced to germs defined on a 1dimensional domain. The appropriate way to deal with the bifurcation of steady states of the original problem is to restrict the extended problem, which possesses a compact Lie group $\Gamma$ of symmetries, to those steady solutions that are invariant under a subgroup $\Sigma$ of $\Gamma$. We formulate this idea more precisely in $\S 2$.

The influence of hidden symmetries on the singularity theory of steady-state bifurcations has so far been studied only in a few special cases, notably [2]. Before moving on to this topic, we set up some notation. Throughout, the word 'germ' refers to a germ defined at the origin. Denote by $h:(V \times \mathbb{R}, 0) \rightarrow W$ a oneparameter smooth germ defined on a finite-dimensional vector space $V$ and taking values on a finite-dimensional vector space $W$. If $h(0,0)=0$, we write $h:(V \times \mathbb{R}, 0) \rightarrow(W, 0)$. For $\Gamma$ a compact Lie group acting linearly on $V$, we say that a one-parameter smooth germ $\widetilde{f}:(V \times \mathbb{R}, 0) \rightarrow \mathbb{R}$ is $\Gamma$-invariant if

$$
\tilde{f}(\gamma x, \lambda)=\tilde{f}(x, \lambda), \quad \text { for all } \gamma \in \Gamma,
$$

and we denote by $\mathscr{E}_{\Gamma}$ the ring of such germs. If $\Gamma$ also acts linearly on $W$, then $\widetilde{g}:(V \times \mathbb{R}, 0) \rightarrow W$ is $\Gamma$-equivariant (or commutes with the action of $\Gamma$ ) if

$$
\widetilde{g}(\gamma x, \lambda)=\gamma \widetilde{g}(x, \lambda), \quad \text { for all } \gamma \in \Gamma .
$$

Most of this paper refers to equivariant germs for which $V=W$, and in this case we assume that the actions of $\Gamma$ on the source and on the target are the same. We assume throughout that $\Gamma$ acts trivially on the parameter space $\mathbb{R}$. Denote by $\overrightarrow{\mathscr{E}}_{\Gamma}$ the module of $\Gamma$-equivariant smooth germs $\widetilde{g}:(V \times \mathbb{R}, 0) \rightarrow V$ over the ring $\mathscr{E}_{\Gamma}$. Finally, define a bifurcation problem on $V$ to be a smooth germ $h:(V \times \mathbb{R}, 0) \rightarrow(V, 0)$ whose derivative $(d h)_{0,0}$ with respect to $x$ at $(0,0)$ is singular.

It is well known (see, for instance, [12]) that the equivariant version of the Liapunov-Schmidt reduction procedure preserves the symmetries of a $\Gamma$-equivariant problem. It therefore reduces the study of steady-state bifurcation of a partial differential equation to the bifurcation of zeros of a germ defined on a finitedimensional vector space. This space is isomorphic to the critical eigenspace (kernel of the linearized operator) provided that this kernel is finite dimensional, and provided that certain other standard technical requirements hold. The essential idea of the Liapunov-Schmidt reduction is that steady-state bifurcations of certain partial differential equations (such as those studied in $[\mathbf{2}, \mathbf{5}, \mathbf{1 4}, \mathbf{8}]$ ) can be projected onto a finite-dimensional vector space. As we discuss in $\S 2$, a bifurcation problem $g$ with hidden symmetries admits a $\Gamma$-equivariant extension $\widetilde{g}$. So throughout this paper, $\widetilde{g}$ is assumed to be a Liapunov-Schmidt reduced germ obtained by the procedure just described.

\section{Hidden symmetries}

The notion of hidden symmetries was first formalized by Golubitsky et al. [11] using slightly different terminology. These authors were mainly motivated by two 
papers of Hunt $[\mathbf{1 5}, \mathbf{1 6}]$ which describe the buckling of a right circular cylinder under end loading in terms of the parabolic umbilic catastrophe. Hunt notes that the parabolic umbilic appears in a context where some less degenerate singularities (such as the elliptic or hyperbolic umbilic) would appear more likely. Golubitsky et al. [11] explain Hunt's result in terms of an extra symmetry that occurs on a particular subspace, and generalize Hunt's procedure. However, they do not attempt to develop the singularity theory for this context, which is the main purpose of this paper.

Let $\Gamma$ be a compact Lie group acting linearly on an $n$-dimensional vector space $V$ and let $\widetilde{g}:(V \times \mathbb{R}, 0) \rightarrow V$ be a $\Gamma$-equivariant germ. For a subgroup $\Sigma \subseteq \Gamma$, recall that the fixed-point subspace of $\Sigma$ is

$$
\text { Fix } \Sigma=\{x \in V: \sigma x=x, \forall \sigma \in \Sigma\} .
$$

The isotropy subgroup of $x_{0} \in V$ is $\Sigma_{x_{0}}=\left\{\sigma \in \Gamma: \sigma x_{0}=x_{0}\right\}$. The normalizer of a subgroup $\Sigma$ in $\Gamma$ is $N(\Sigma)=\left\{\gamma \in \Gamma: \gamma^{-1} \Sigma \gamma=\Sigma\right\}$, and this is a subgroup of $\Gamma$ that contains $\Sigma$.

We are interested in zeros of $\widetilde{g}:(V \times \mathbb{R}, 0) \rightarrow V$ that are invariant under the action of a subgroup $\Sigma$ of $\Gamma$. By equivariance we have $\widetilde{g}(\operatorname{Fix} \Sigma \times \mathbb{R}) \subseteq$ Fix $\Sigma$. Therefore, in order to find $\Sigma$-invariant solutions to $\widetilde{g}(x, \lambda)=0$, we can restrict the domain to Fix $\Sigma$ and find zeros there. We therefore study zeros of the germ

$$
g:(\text { Fix } \Sigma \times \mathbb{R}, 0) \rightarrow \text { Fix } \Sigma
$$

defined to be the restriction of $\widetilde{g}$ to Fix $\Sigma \times \mathbb{R}$.

REMARK 2.1. Most classifications in the literature have been performed in the context of $\Gamma$-equivariant bifurcation problems $\widetilde{g}:(V \times \mathbb{R}, 0) \rightarrow V$, when the group of symmetries $\Gamma$ satisfies Fix $\Gamma=\{0\}$. Many useful results derive from this property; for example, this is one of the hypotheses of Theorem XIII.3.5 of [13] which implies the Equivariant Branching Lemma [13, Theorem XIII.3.3], one of the most important existence theorems for solutions to equivariant bifurcation problems. We similarly assume that Fix $\Gamma=0$. In fact, we assume that, for all $\lambda$,

$$
g(0, \lambda) \equiv 0 \text {. }
$$

Both conditions are equivalent since $g(0, \lambda)=0$ if and only if $\widetilde{g}(0, \lambda)=0$, and $\widetilde{g}(0, \lambda)=0$ if and only if Fix $\Gamma=\{0\}$; see [13]. As we discuss later, assumption (2.0.3) implies that the group of L-contact equivalences (Definition 3.2) is a geometric subgroup in the sense of Damon [6]. It will become clear that (2.0.3) is not a necessary assumption when $L \equiv 0$ (that is, when we are interested in the classification of germs with trivial linearization at the origin), but for convenience we assume it throughout.

We now turn to hidden symmetries. The normalizer $N(\Sigma)$ leaves Fix $\Sigma$ invariant (and when $\Sigma$ is an isotropy subgroup of $\Gamma$, then $N(\Sigma)$ is the largest subgroup of $\Gamma$ with this property). For this reason, elements in $N(\Sigma)$ are called the apparent symmetries in Fix $\Sigma$, the domain of the problem. Now, the existence of the extension $\widetilde{g}$ of $g$ implies that not all symmetries in the equation $g=0$ are so obvious as the symmetries in $N(\Sigma)$. The idea is as follows. Let $\gamma \in \Gamma$ and suppose that $\gamma$ does not leave Fix $\Sigma$ invariant (so $\gamma \notin N(\Sigma)$ ), but

$$
(\gamma \cdot \operatorname{Fix} \Sigma) \cap \operatorname{Fix} \Sigma \neq\{0\} .
$$


Then there exists a non-trivial $x \in$ Fix $\Sigma$ such that $\gamma x \in$ Fix $\Sigma$. Hence $\widetilde{g}(x, \lambda), \widetilde{g}(\gamma x, \lambda) \in$ Fix $\Sigma$. But these are just $g(x, \lambda)$ and $g(\gamma x, \lambda)$ respectively. Since $\widetilde{g}$ is $\Gamma$-equivariant, it follows that

$$
g(\gamma x, \lambda)=\gamma g(x, \lambda) .
$$

Therefore the symmetry $\gamma$ of $\widetilde{g}$ places an extra condition on $g$, in addition to those conditions imposed by the apparent symmetries in $N(\Sigma)$. In fact, it is straightforward to see that $g$ is $N(\Sigma)$-equivariant, but the discussion above implies that the elements in $N(\Sigma)$ are not all the symmetries to be taken into account to ensure that $g$ can be extended to a $\Gamma$-equivariant $\widetilde{g}$. We call the extra element $\gamma$ satisfying (2.0.4) a hidden symmetry for $g$. The precise definition is as follows.

Definition 2.2. Let $g:($ Fix $\Sigma \times \mathbb{R}, 0) \rightarrow$ Fix $\Sigma$ be a one-parameter smooth germ extendible to a $\Gamma$-equivariant smooth germ $\widetilde{g}:(V \times \mathbb{R}, 0) \rightarrow V$, where $V$ is a finite-dimensional vector space. A hidden symmetry of $g$ is a non-zero element $\gamma$ in $\Gamma$ such that $\gamma$ does not leave the whole subspace Fix $\Sigma$ invariant, but $(\gamma \cdot \operatorname{Fix} \Sigma) \cap \operatorname{Fix} \Sigma \neq\{0\}$.

The following proposition describes one case where the existence of a $\Gamma$ equivariant extension does not imply any extra symmetry in the original problem.

Proposition 2.3. Hidden symmetries do not occur when $\Sigma$ is a maximal isotropy subgroup.

Proof. Suppose that $\gamma \in \Gamma$ is a hidden symmetry. It is easy to see that $W=(\gamma \cdot$ Fix $\Sigma) \cap$ Fix $\Sigma$ is itself a fixed-point subspace, namely the fixed-point subspace of the subgroup $\widetilde{\Sigma}$ generated by $\Sigma$ and $\gamma \Sigma \gamma^{-1}$. Since $\gamma \notin N(\Sigma)$, then $W$ is a proper subspace of Fix $\Sigma$. By assumption, there exists $x \in W$ with $x \neq 0$. Since $\Sigma \subseteq \Sigma_{x}$ and $\Sigma$ is maximal, we get $\Sigma=\Sigma_{x}$. Also, $x \in W=$ Fix $\widetilde{\Sigma}$, so $\widetilde{\Sigma} \subseteq \Sigma_{x}$. Hence, $\widetilde{\Sigma} \subseteq \Sigma$. Therefore, Fix $\Sigma \subseteq$ Fix $\widetilde{\Sigma}=W$, a contradiction.

REMARK 2.4. The absence of hidden symmetries does not imply that every smooth $N(\Sigma)$-equivariant $g$ on Fix $\Sigma$ extends to a $\Gamma$-equivariant $\widetilde{g}$ on $V$, even if $\Sigma$ is a maximal isotropy subgroup. The standard example is $\Gamma=\mathbf{D}_{5}$ in its standard action on $\mathbb{R}^{2} \equiv \mathbb{C}$. This example is described in [13, Exercise XII.4.11, p. 49], but there is a typographical error, with $x^{3}$ in place of $x^{2}$. We therefore sketch the reasoning. The $\Gamma$-equivariant mappings take the form

$$
\widetilde{g}(z)=p\left(z \bar{z}, \operatorname{Re}\left(z^{5}\right)\right) z+q\left(z \bar{z}, \operatorname{Re}\left(z^{5}\right)\right) \bar{z}^{4},
$$

for a coordinate $z$ in $\mathbb{C}$ and smooth $p$ and $q$. The restriction of $\widetilde{g}$ to $\mathbb{R} \times\{0\}=$ Fix $\mathbf{Z}_{2}^{\kappa}$ is

$$
g(z)=p\left(x^{2}, x^{5}\right) x+q\left(x^{2}, x^{5}\right) x^{4}
$$

whose Taylor expansion (jet) lacks the term $x^{2}$. Here $\kappa \cdot z=\bar{z}$. The group $\Sigma=\mathbf{Z}_{2}^{\kappa}$ is a maximal isotropy subgroup with normalizer $N(\Sigma)=\Sigma$, acting trivially on Fix $\Sigma$. So every smooth function of $x$ is an $N(\Sigma)$-equivariant. Therefore the polynomial $N(\Sigma)$-equivariant $h(x)=x^{2}$ does not extend to a smooth $\mathbf{D}_{5}$-equivariant.

The obstacle is smoothness: by Tychonoff's theorem and averaging over $\mathbf{D}_{5}$, the map $h$ extends to a continuous $\mathbf{D}_{5}$-equivariant. 
We end this section by setting up some further notation. Let $m$ denote the dimension of Fix $\Sigma, x$ the coordinates of Fix $\Sigma$, and $\lambda$ the distinguished parameter. It is well known (see [13]) that the $\Gamma$-invariants form a ring and the $\Gamma$-equivariants form a module over this ring. What we need here is a variation of this result. We have a subgroup $\Sigma$ of $\Gamma$ and we want to consider the restrictions of the $\Gamma$ invariants and $\Gamma$-equivariants to Fix $\Sigma \times \mathbb{R}$. It remains true that the restricted invariant germs form a ring, and the restricted equivariant germs form a module over this ring.

Let

$$
\mathscr{E}_{\Gamma}(\operatorname{Fix} \Sigma)=\left\{f:(\operatorname{Fix} \Sigma \times \mathbb{R}, 0) \rightarrow \mathbb{R}: f=\left.\tilde{f}\right|_{\text {Fix } \Sigma \times \mathbb{R}} \text { for some } \tilde{f} \in \mathscr{E}_{\Gamma}\right\} .
$$

Schwarz [22] proves that there is a finite set of polynomials generating the ring of $\Gamma$-invariant smooth germs, in the sense that any $\Gamma$-invariant smooth germ is the germ of a smooth function of those generators, so it also follows that there exists a finite set of polynomials generating $\mathscr{E}_{\Gamma}(\operatorname{Fix} \Sigma)$. We shall denote by $\mathscr{M}$ the maximal ideal in $\mathscr{E}_{\Gamma}($ Fix $\Sigma)$ of germs that vanish at the origin. Also, let $\overrightarrow{\mathscr{E}}_{\Gamma}($ Fix $\Sigma)$ denote the module over $\mathscr{E}_{\Gamma}($ Fix $\Sigma)$ of $\Gamma$-equivariant smooth map germs on $V \times \mathbb{R}$ when restricted to Fix $\Sigma \times \mathbb{R}$. Notice that, since Fix $\Gamma=\{0\}$, any $g \in \overrightarrow{\mathscr{E}}_{\Gamma}($ Fix $\Sigma)$ satisfies $g(0, \lambda)=0$, for all $\lambda$, so we define

$$
\overrightarrow{\mathscr{E}}_{\Gamma}(\operatorname{Fix} \Sigma)=\left\{g:(\text { Fix } \Sigma \times \mathbb{R}, 0) \rightarrow(\text { Fix } \Sigma, 0): g=\left.\widetilde{g}\right|_{\text {Fix } \Sigma \times \mathbb{R}} \text { for some } \widetilde{g} \in \widetilde{\mathscr{E}}_{\Gamma}\right\}
$$

By Poènaru's theorem [21], there exists a finite set of polynomials generating the module of $\Gamma$-equivariant smooth germs over the ring $\mathscr{E}_{\Gamma}$, so it also follows that there exists a finite number of generators with polynomial entries for the module $\overrightarrow{\mathscr{E}}_{\Gamma}($ Fix $\Sigma)$ over the ring $\mathscr{E}_{\Gamma}(\mathrm{Fix} \Sigma)$. Finally, let $\mathscr{E}_{\lambda}$ denote the ring of function germs depending only on $\lambda$, and let $\mathscr{M}_{\lambda}$ denote the ideal of germs in $\mathscr{E}_{\lambda}$ that vanish at the origin.

\section{Singularity theory}

The aim of this section is to set up a singularity-theoretic approach for the classification of symmetric bifurcation problems that combine two types of constraint: hidden symmetries, and restrictions on the linearization. We adapt results from singularity theory found mainly in $[4,6,10,13]$, and throughout this section we assume some familiarity with these papers.

\subsection{The equivalence relation}

In this subsection we define an appropriate equivalence relation for the classification of bifurcation problems with hidden symmetries whose linearization at the origin is a fixed singular matrix $L$, not necessarily the zero matrix. The definition is given in $\S 3.1 .2$. First we make a remark concerning the group of contact equivalences motivating the definition of the module $\overleftrightarrow{\mathscr{E}}_{\Gamma}($ Fix $\Sigma)$ of families of diffeomorphisms that we use to define the $L$-equivalence relation; see expression (3.1.4).

\subsubsection{Matrix germs in the classification}

Recall the definition of Mather's group of contact equivalences [18]. Let $\mathscr{C}$ be the group of diffeomorphism germs $H$ on $\mathbb{R}^{n} \times \mathbb{R}^{p}$ which leave fixed the projection on $\mathbb{R}^{n}$ and preserve the subspace $\mathbb{R}^{n} \times\{0\}$. Any $H \in \mathscr{C}$ is of the form $H(x, y)=\left(x, H_{1}(x, y)\right)$ where $H_{1}:\left(\mathbb{R}^{n} \times \mathbb{R}^{p}, 0\right) \rightarrow\left(\mathbb{R}^{p}, 0\right)$ satisfies $H_{1}(x, 0)=0$. 
Note that $\mathscr{C}$ acts on the set of map germs $g:\left(\mathbb{R}^{n}, 0\right) \rightarrow\left(\mathbb{R}^{p}, 0\right)$ by $H \cdot g(x)=H_{1}(x, g(x))$. Let $\mathscr{R}$ denote the group of diffeomorphism germs on $\mathbb{R}^{n}$. The group of contact equivalences is the semidirect product $\mathscr{K}=\mathscr{R} \cdot \mathscr{C}$. It is well known [18] that the orbits of $\mathscr{C}$ coincide with the orbits of that subgroup for which the germs $H_{1}$ are families of matrix-valued germs. This property also holds for the equivariant case, and we state this result as it appears in [13].

Proposition 3.1. Let $Q:(V \times \mathbb{R} \times V, 0) \rightarrow(V, 0)$ be a parametrised family of diffeomorphism germs; that is, for each $(x, \lambda) \in V \times \mathbb{R}, Q(x, \lambda, \cdot)$ is a diffeomorphism germ on V. If $Q(\gamma x, \lambda, \gamma y)=\gamma Q(x, \lambda, y)$, for all $\gamma \in \Gamma$, then for any $\Gamma$-equivariant germ $\tilde{g}:(V \times \mathbb{R}, 0) \rightarrow V$ we have

$$
Q(x, \lambda, \widetilde{g}(x, \lambda))=\widetilde{S}(x, \lambda) \widetilde{g}(x, \lambda),
$$

where $\widetilde{S}$ is a smooth matrix-valued germ, $\widetilde{S}(0,0)$ is an invertible matrix, and $\widetilde{S}(\gamma x, \lambda) \gamma y=\gamma \widetilde{S}(x, \lambda)$, for all $\gamma \in \Gamma$.

\section{Proof. See [13, Proposition XIV.1.5].}

Once again, as discussed in $\S 2$ for the definition of a problem with hidden symmetry, the appropriate way to define an equivalence relation in this context is to make explicit the existence of a $\Gamma$-equivariant extension. In other words, from the symmetry imposed on the bifurcation problems in our context, we consider the families of diffeomorphisms on Fix $\Sigma \times \mathbb{R}$ arising as matrix germs $S:($ Fix $\Sigma \times \mathbb{R}, 0) \rightarrow$ $\operatorname{Hom}(\operatorname{Fix} \Sigma$, Fix $\Sigma)$ corresponding to matrix germs $\widetilde{S}:(V \times \mathbb{R}, 0) \rightarrow \operatorname{Hom}(V, V)$ as given in Proposition 3.1. To accomplish this, consider the action of $\Gamma$ on $\operatorname{Hom}(V, V)$ defined by

$$
\gamma \cdot M=\gamma M \gamma^{-1}
$$

and let

$$
\widetilde{S}:(V \times \mathbb{R}, 0) \rightarrow \operatorname{Hom}(V, V)
$$

be a $\Gamma$-equivariant smooth matrix-valued germ, that is,

$$
\widetilde{S}(\gamma x, \lambda) \gamma=\gamma \widetilde{S}(x, \lambda) \quad \text { for all } \gamma \in \Gamma .
$$

Denote by $\overleftrightarrow{\mathscr{E}}_{\Gamma}$ the module of germs (3.1.1) satisfying (3.1.2). We now consider the matrix-valued germs on Fix $\Sigma \times \mathbb{R}$ :

$$
S:(\operatorname{Fix} \Sigma \times \mathbb{R}, 0) \rightarrow \operatorname{Hom}(\operatorname{Fix} \Sigma, \text { Fix } \Sigma)
$$

given by

$$
S(x, \lambda)=\left.\widetilde{S}(x, \lambda)\right|_{\text {Fix } \Sigma} \text { for all } x \in \text { Fix } \Sigma,
$$

where $\widetilde{S} \in \overleftrightarrow{\mathscr{E}}_{\Gamma}$. We denote $\overleftrightarrow{\mathscr{E}}_{\Gamma}($ Fix $\Sigma)$ the module of such matrix-valued germs, that is,

$$
\begin{aligned}
& \overleftrightarrow{\mathscr{E}}_{\Gamma}(\text { Fix } \Sigma)=\{S:(\text { Fix } \Sigma \times \mathbb{R}, 0) \rightarrow \operatorname{Hom}(\text { Fix } \Sigma, \text { Fix } \Sigma): \\
& \left.\qquad(x, \lambda)=\left.\widetilde{S}(x, \lambda)\right|_{\text {Fix } \Sigma} \text { for some } \widetilde{S} \in \overleftrightarrow{\mathscr{E}}_{\Gamma}\right\}
\end{aligned}
$$

Poènaru's theorem [21] implies that $\overleftrightarrow{\mathscr{E}}_{\Gamma}($ Fix $\Sigma)$ is a finitely generated module over the ring $\mathscr{E}_{\Gamma}($ Fix $\Sigma)$. 


\subsubsection{The appropriate equivalence relation}

In the classification and recognition of bifurcation problems we are interested in preserving various properties of the associated germs under some equivalence relation. For instance, when symmetry is present, the equivalence relation is required to preserve this symmetry. In our case, the germs are defined on Fix $\Sigma$ and admit a $\Gamma$-equivariant extension to $V$. In addition, for a particular singular matrix $L$, we want to classify germs $g$ such that $(d g)_{0,0}=L$, so we require the equivalence relation to preserve this matrix too.

Definition 3.2. Let $L$ be a fixed but arbitrary $m \times m$ singular matrix. Let $g$ be a bifurcation problem in $\overrightarrow{\mathscr{E}}_{\Gamma}($ Fix $\Sigma)$. We say that $h \in \overrightarrow{\mathscr{E}}_{\Gamma}($ Fix $\Sigma)$ is L-contact equivalent to $g$, or simply equivalent to $g$, if there exist $S \in \overleftrightarrow{\mathscr{E}}_{\Gamma}($ Fix $\Sigma)$, $\Phi \in \overrightarrow{\mathscr{E}}_{\Gamma}($ Fix $\Sigma)$ and $\Lambda \in \mathscr{E}_{\lambda}$ such that

$$
h(x, \lambda)=S(x, \lambda) g(\Phi(x, \lambda), \Lambda(\lambda)),
$$

where

(i) $\Phi(0,0)=0$,

(ii) $\Lambda(0)=0$ and $\Lambda^{\prime}(0)>0$,

(iii) $S(0,0)$ and $(d \Phi)_{0,0}$ are invertible matrices in the same connected component as the identity in $\mathbf{G L}(m)$,

(iv) $S(0,0) L(d \Phi)_{0,0}=L$.

When $\Lambda(\lambda)=\lambda$ we say that $h$ is $L$-strongly equivalent to $g$.

REMARK 3.3. 1. This definition applies to all $g \in \overleftrightarrow{\mathscr{E}}_{\Gamma}($ Fix $\Sigma)$, whether or not $(d g)_{0,0}=L$. However, note that if $(d h)_{0,0}=L$ and $h_{1}$ is $L$-contact equivalent to $h$, then $\left(d h_{1}\right)_{0,0}=L$.

2. As we mentioned in $\S 2$ we always assume Fix $\Gamma=\{0\}$, so condition (i) is automatically satisfied. The assumptions that $S(0,0)$ and $(d \Phi)_{0,0}$ are in the connected component of the identity, and that $\Lambda^{\prime}(0)>0$, are stability-preserving conditions.

Denote by $\mathscr{K}_{L}$ the group of equivalences defined above:

$\mathscr{K}_{L}=\left\{(S, \Phi, \Lambda) \in \overleftrightarrow{\mathscr{E}}_{\Gamma}(\operatorname{Fix} \Sigma) \times \overleftrightarrow{\mathscr{E}}_{\Gamma}(\operatorname{Fix} \Sigma) \times \mathscr{E}_{\lambda}\right.$ :

$S, \Phi, \Lambda$ satisfy conditions (i)-(iv) $\}$.

Writing $\varphi_{i}=\left(\Phi_{i}, \Lambda_{i}\right)$, for $i=1,2$, we see that the action of $\mathscr{K}_{L}$ on $\overleftrightarrow{\mathscr{E}}_{\Gamma}($ Fix $\Sigma)$ is induced by group multiplication $\left(S_{2}, \varphi_{2}\right) \cdot\left(S_{1}, \varphi_{1}\right)=\left(S_{2} \cdot\left(S_{1} \circ \varphi_{2}\right), \varphi_{1} \circ \varphi_{2}\right)$. When $h$ is $L$-contact equivalent to $g$ we write $h \sim_{\mathscr{K}_{L}} g$, or simply $h \sim g$ when the group $\mathscr{K}_{L}$ of equivalences is clear from the context.

Although the action of $\mathscr{K}_{L}$ is defined on the whole space $\overrightarrow{\mathscr{E}}_{\Gamma}($ Fix $\Sigma)$, in practice we are mainly interested in the orbit $\mathscr{K}_{L} \cdot g$ under $\mathscr{K}_{L}$ of a germ $g$ in the affine subspace

$$
\overrightarrow{\mathscr{E}}_{\Gamma} L(\operatorname{Fix} \Sigma)=\left\{g \in \overrightarrow{\mathscr{E}}_{\Gamma}(\text { Fix } \Sigma):(d g)_{0,0}=L\right\} .
$$

In fact, our intention is to classify such germs, and this is what motivates condition (iv) of Definition 3.2. If we also use the notation $\mathscr{K}$ for the group of contact equivalences $(S, \Phi, \Lambda)$ satisfying (i), (ii), (iii), then it is straightforward to show that $\mathscr{K}_{L}$ is a subgroup of $\mathscr{K}$. Moreover, the orbits of any $g \in \overrightarrow{\mathscr{E}}_{\Gamma}^{L}(\operatorname{Fix} \Sigma)$ under $\mathscr{K}_{L}$ and $\mathscr{K}$ satisfy

$$
\mathscr{K}_{L} \cdot g=\mathscr{K} \cdot g \cap \overrightarrow{\mathscr{E}}_{\Gamma}^{L}(\operatorname{Fix} \Sigma) .
$$


We finish this subsection with an example of an $L$-contact equivalence relation when the linearization $L$ is nilpotent. This equivalence relation is considered in $\S 4.4$.

EXAMPLE 3.4. Let $\Gamma=\mathbf{O}(2)$, the orthogonal group in two dimensions, and consider its action on $\mathbb{C}^{2}$ generated by $\theta \cdot\left(z_{1}, z_{2}\right)=\left(e^{\theta i} z_{1}, e^{\theta i} z_{2}\right)$, for $\theta \in S^{1}$, and $\kappa \cdot\left(z_{1}, z_{2}\right)=\left(\bar{z}_{1}, \bar{z}_{2}\right)$. Here $\theta$ is rotation by angle $\theta$ and $\kappa$ is the 'flip', a reflection (see [13, §XXa]). Take $\Sigma=\mathbf{Z}_{2}$, the subgroup generated by $\kappa$. Then Fix $\mathbf{Z}_{2}=\mathbb{R}^{2}$. As we see in $\S 4.4 .1$, a general element in $\overrightarrow{\mathscr{E}}_{\mathbf{O}(2)}\left(\right.$ Fix $\left.\mathbf{Z}_{2}\right)$ is of the form

$$
g(x, y, \lambda)=(a(u, v, \lambda) x+b(u, v, \lambda) y, c(u, v, \lambda) y+d(u, v, \lambda) x),
$$

where $u=x^{2}$ and $v=y^{2}$. We refer to (4.4.6) for generators of $\overleftrightarrow{\mathscr{E}}_{\mathbf{O}(2)}\left(\right.$ Fix $\left.\mathbf{Z}_{2}\right)$. We want to classify bifurcation problems $g$ such that $(d g)_{0,0}$ is given by

$$
L=\left(\begin{array}{ll}
0 & 1 \\
0 & 0
\end{array}\right) \text {. }
$$

A germ $h$ is $L$-contact equivalent to $g$ if and only if

$$
h(x, y, \lambda)=S(x, y, \lambda) g(\Phi(x, y, \lambda), \Lambda(\lambda)),
$$

where $S \in \overleftrightarrow{\mathscr{E}}_{\mathbf{O}(2)}\left(\operatorname{Fix} \mathbf{Z}_{2}\right), \Phi \in \overrightarrow{\mathscr{E}}_{\mathbf{O}(2)}\left(\operatorname{Fix} \mathbf{Z}_{2}\right)$ and $\Lambda \in \mathscr{E}_{\lambda}$ satisfy

$$
\begin{gathered}
S(0,0,0)=\left(\begin{array}{cc}
\alpha_{0} & \beta_{0} \\
0 & \delta_{0}
\end{array}\right), \quad \alpha_{0} \delta_{0}>0, \\
(d \Phi)_{0,0,0}=\left(\begin{array}{cc}
A_{0} & B_{0} \\
0 & C_{0}
\end{array}\right), \quad A_{0} C_{0}>0, \\
\Lambda(0)=0, \quad \Lambda^{\prime}(0)>0,
\end{gathered}
$$

and

$$
\alpha_{0} C_{0}=1 .
$$

The entries that vanish in the matrices (3.1.9) and (3.1.10) and the condition (3.1.12) are consequences of the condition (iv) in Definition 3.2. In $\S 4.4$ we classify germs (3.1.8) under the equivalence relation defined above as an example of the classification of a singularity with non-trivial linearization.

\subsection{Tangent spaces and unfoldings}

Here we give some definitions that will be used in the next subsections. We also present Lemma 3.11, a result that follows from Damon's determinacy theorem [6]. This lemma implies that a finite codimension bifurcation problem is equivalent to its Taylor polynomial of order $k$ for some $k$.

For $\alpha \in \mathbb{R}^{k}$, for some $k$, let us denote by $\mathscr{E}_{\Gamma}^{\alpha}(\operatorname{Fix} \Sigma)$ the ring of $k$-parameter families of germs in $\mathscr{E}_{\Gamma}(\operatorname{Fix} \Sigma)$, and by $\overrightarrow{\mathscr{E}}_{\Gamma}^{\alpha}(\operatorname{Fix} \Sigma)$ the $\mathscr{E}_{\Gamma}^{\alpha}(\operatorname{Fix} \Sigma)$-module of $k$ parameter families of germs in $\overrightarrow{\mathscr{E}}_{\Gamma}(\mathrm{Fix} \Sigma)$. Consider also analogous definitions for the module $\overleftrightarrow{\mathscr{E}}_{\Gamma}^{\alpha}(\mathrm{Fix} \Sigma)$ and the ring $\mathscr{E}_{\lambda}^{\alpha}$ of $k$-parameter families of germs in $\overleftrightarrow{\mathscr{E}}_{\Gamma}($ Fix $\Sigma)$ and $\mathscr{E}_{\lambda}$ respectively.

Definition 3.5. For $\alpha \in \mathbb{R}^{k}$, for some $k$, a k-parameter unfolding of a germ $g \in \overrightarrow{\mathscr{E}}_{\Gamma}($ Fix $\Sigma)$ is a germ $G \in \overrightarrow{\mathscr{E}}_{\Gamma}^{\alpha}($ Fix $\Sigma)$ such that $G(x, \lambda, 0)=g(x, \lambda)$. 
Definition 3.6. For $\alpha \in \mathbb{R}^{k}$ and $\beta \in \mathbb{R}^{l}$, if $H \in \overrightarrow{\mathscr{E}}_{\Gamma}^{\beta}($ Fix $\Sigma)$ is an l-parameter unfolding of $g$ and $G \in \overrightarrow{\mathscr{E}}_{\Gamma}^{\alpha}($ Fix $\Sigma)$ is a $k$-parameter unfolding of $g$, we say that $H(x, \lambda, \beta)$ factors through $G(x, \lambda, \alpha)$ if

$$
H(x, \lambda, \beta)=S(x, \lambda, \beta) G(\Phi(x, \lambda, \beta), \Lambda(\lambda, \beta), A(\beta))
$$

where

$$
\begin{aligned}
& S \in \overleftrightarrow{\mathscr{E}}_{\Gamma}^{\beta}(\operatorname{Fix} \Sigma), \quad S(x, \lambda, 0)=I, \\
& \Phi \in \overrightarrow{\mathscr{E}}_{\Gamma}^{\beta}(\operatorname{Fix} \Sigma), \quad \Phi(x, \lambda, 0)=x, \\
& \Lambda(\lambda, 0)=\lambda, \\
& A(0)=0 .
\end{aligned}
$$

Definition 3.7. An unfolding $G$ of $g$ is versal if every unfolding $H$ of $g$ factors through $G$. It is universal if the number of parameters is minimal. This number is called the codimension $\operatorname{cod} g$ of $g$.

For $\alpha \in \mathbb{R}^{k}$, let $\mathscr{E}_{\alpha, \alpha}$ denote the ring of germs at the origin $A:\left(\mathbb{R}^{k}, 0\right) \rightarrow \mathbb{R}^{k}$. We now define the group $\mathscr{K}_{L}^{u}(k)$ of equivalences for $k$-parameter unfoldings. This is an extension of the group $\mathscr{K}_{L}$ in the following way:

$$
\begin{aligned}
\mathscr{K}_{L}^{u}(k)=\left\{(S, \Phi, \Lambda, A) \in \overleftrightarrow{\mathscr{E}}_{\Gamma}^{\alpha}(\operatorname{Fix} \Sigma) \times \overrightarrow{\mathscr{E}}_{\Gamma}^{\alpha}(\operatorname{Fix} \Sigma) \times \mathscr{E}_{\lambda}^{\alpha} \times \mathscr{E}_{\alpha, \alpha}:\right. \\
(S(\cdot, \cdot, 0), \Phi(\cdot, \cdot, 0), \Lambda(\cdot, 0)) \in \mathscr{K}_{L} \text {, and }
\end{aligned}
$$

$A$ is a germ of diffeomorphism such that $A(0)=0\}$.

The tangent space to $\mathscr{K}_{L}$ at the identity

$$
\mathbf{1}=(S(x, \lambda) \equiv I, \Phi(x, \lambda) \equiv x, \Lambda(\lambda) \equiv \lambda)
$$

is defined by

$$
\mathscr{T}\left(\mathscr{K}_{L}\right)=\left\{\left.\frac{d}{d t} \delta_{t}\right|_{t=0}: \delta_{t} \in \mathscr{K}_{L}, \delta_{0}=\mathbf{1}\right\} .
$$

A straightforward calculation yields

$$
\begin{aligned}
& \mathscr{T}\left(\mathscr{K}_{L}\right)=\left\{(S, \Phi, \Lambda) \in \overleftrightarrow{\mathscr{E}}_{\Gamma}(\mathrm{Fix} \Sigma) \times \overrightarrow{\mathscr{E}}_{\Gamma}(\mathrm{Fix} \Sigma) \times \mathscr{E}_{\lambda}:\right. \\
& \left.\Phi(0,0)=0, \Lambda(0)=0, S(0,0) L+L(d \Phi)_{0,0}=0\right\}
\end{aligned}
$$

We now define the extended tangent space $\mathrm{T}\left(\mathscr{K}_{L}\right)$ of $\mathscr{K}_{L}$. A general element in $\mathrm{T}\left(\mathscr{K}_{L}\right)$ is defined by

$$
\left.\frac{d}{d t} \varepsilon_{t}\right|_{t=0},
$$

where $\varepsilon_{t} \in \mathscr{K}_{L}^{u}(1)$ is a one-parameter unfolding of the identity, so

$$
\mathrm{T}\left(\mathscr{K}_{L}\right)=\left\{(S, \Phi, \Lambda): S \in \overleftrightarrow{\mathscr{E}}_{\Gamma}(\operatorname{Fix} \Sigma), \Phi \in \overrightarrow{\mathscr{E}}_{\Gamma}(\operatorname{Fix} \Sigma), \Lambda \in \mathscr{E}_{\lambda}\right\}
$$

We now turn to the definitions of tangent spaces of a germ. As for the expression (3.2.4) of $\mathscr{T}\left(\mathscr{K}_{L}\right)$, we shall see that the definitions of the tangent space $\mathscr{T}(g)$ and the restricted tangent space $\mathrm{RT}(g)$ of a germ $g$ are derived from $L$-contact equivalence, so in cases where $(d g)_{0,0}=L \neq 0$, the algebraic expressions of 
$\mathscr{T}(g)$ and $\mathrm{RT}(g)$ depend on $L$. On the other hand, whatever the linearization, we want to define the extended tangent space $\mathrm{T}(g)$ in a natural way so that we can find a universal unfolding of $g$ by computing a complement to $\mathrm{T}(g)$ in $\overrightarrow{\mathscr{E}}_{\Gamma}(\mathrm{Fix} \Sigma)$. Then cod $g$ coincides with the codimension of $\mathrm{T}(g)$ in $\overrightarrow{\mathscr{E}}_{\Gamma}($ Fix $\Sigma)$.

Define the tangent space of a germ $g \in \overrightarrow{\mathscr{E}}_{\Gamma}($ Fix $\Sigma)$ to be

$$
\mathscr{T}(g)=\left\{\left.\frac{d}{d t}\left(\delta_{t} \cdot g\right)\right|_{t=0}: \delta_{t} \in \mathscr{K}_{L}, \delta_{0}=\mathbf{1}\right\} .
$$

Hence,

$$
\begin{gathered}
\mathscr{T}(g)=\left\{S g+(d g) \Phi: S \in \overleftrightarrow{\mathscr{E}}_{\Gamma}(\operatorname{Fix} \Sigma), \Phi \in \overrightarrow{\mathscr{E}}_{\Gamma}(\operatorname{Fix} \Sigma), \Phi(0,0)=0,\right. \\
\left.S(0,0) L+L(d \Phi)_{0,0}=0\right\}+\mathscr{M}_{\lambda} g_{\lambda} .
\end{gathered}
$$

Let $p \in \overrightarrow{\mathscr{E}}_{\Gamma}($ Fix $\Sigma)$ and consider the one-parameter unfolding $g+t p$ of $g$. Suppose that $G(x, \lambda, \alpha)$ is a versal unfolding of $g$. Then $g+t p$ factors through $G$, that is,

$$
g+t p=S(x, \lambda, t) G(\Phi(x, \lambda, t), \Lambda(\lambda, t), A(t))
$$

with $S, \Phi, \Lambda$ and $A$ as in (3.2.2). Differentiate (3.2.7) with respect to $t$ and set $t=0$ :

$$
\begin{aligned}
p(x, \lambda)= & {\left[\dot{S}(x, \lambda, 0) g(x, \lambda)+(d g)_{x, \lambda} \dot{\Phi}(x, \lambda, 0)+g_{\lambda}(x, \lambda) \dot{\Lambda}(\lambda, 0)\right] } \\
& +\sum_{j=1}^{k} G_{\alpha_{j}}(x, \lambda, 0) \dot{A}_{j}(0) .
\end{aligned}
$$

Now define the extended tangent space $\mathrm{T}(g)$ of $g$ to be

$$
\mathrm{T}(g)=\left\{S g+(d g) \Phi: S \in \overleftrightarrow{\mathscr{E}}_{\Gamma}(\operatorname{Fix} \Sigma), \Phi \in \overrightarrow{\mathscr{E}}_{\Gamma}(\operatorname{Fix} \Sigma)\right\}+\mathscr{E}_{\lambda} \cdot g_{\lambda}
$$

Then

Therefore

$$
p(x, \lambda) \in \mathrm{T}(g)+\mathbb{R}\left\{G_{\alpha_{1}}(x, \lambda, 0), \ldots, G_{\alpha_{k}}(x, \lambda, 0)\right\} .
$$

$$
\overrightarrow{\mathscr{E}}_{\Gamma}(\mathrm{Fix} \Sigma)=\mathrm{T}(g)+\mathbb{R}\left\{G_{\alpha_{1}}(x, \lambda, 0), \ldots, G_{\alpha_{k}}(x, \lambda, 0)\right\}
$$

Following the usual singularity-theoretic approach to imperfect bifurcations, we define the restricted tangent space $\mathrm{RT}(g)$ of $g \in \overrightarrow{\mathscr{E}}_{\Gamma}(\mathrm{Fix} \Sigma)$ to be the set of perturbations $p \in \overrightarrow{\mathscr{E}}_{\Gamma}(\mathrm{Fix} \Sigma)$ such that $g+t p$ is $L$-strongly equivalent to $g$ for all small $t$. That is,

$$
g(x, \lambda)+t p(x, \lambda)=S(x, \lambda, t) g(\Phi(x, \lambda, t), \lambda),
$$

where $\quad(S(\cdot, \cdot, t), \Phi(\cdot, \cdot, t), \lambda) \in \mathscr{K}_{L}, \quad S(x, \lambda, 0)=I \quad$ and $\quad \Phi(x, \lambda, 0)=x$. Differentiating (3.2.10) with respect to $t$ at $t=0$, we get

$$
p(x, \lambda)=\dot{S}(x, \lambda, 0) g(x, \lambda)+(d g)_{x, \lambda} \dot{\Phi}(x, \lambda, 0) .
$$

It is easy to see that since $S$ belongs to $\overleftrightarrow{\mathscr{E}}_{\Gamma}(\operatorname{Fix} \Sigma)$, then so does $\dot{S}(\cdot, \cdot, 0)$. Also, since $\Phi$ belongs to $\overrightarrow{\mathscr{E}}_{\Gamma}($ Fix $\Sigma)$, then so does $\dot{\Phi}(\cdot, \cdot, 0)$. Differentiating both sides of the equality $S(0,0, t) L(d \Phi)_{0,0, t}=L$ with respect to $t$ at $t=0$ leads to

$$
\dot{S}(0,0,0) L+L(d \dot{\Phi})_{0,0,0}=0 .
$$


By abuse of notation, we rename $\dot{S}(x, \lambda, 0)$ by $S(x, \lambda)$ and $\dot{\Phi}(x, \lambda, 0)$ by $\Phi(x, \lambda)$ in (3.2.11), and define the restricted tangent space of $g$ to be

$$
\mathrm{RT}(g)=\left\{S g+(d g) \Phi: S \in \overleftrightarrow{\mathscr{E}}_{\Gamma}(\operatorname{Fix} \Sigma), \Phi \in \overrightarrow{\mathscr{E}}_{\Gamma}(\operatorname{Fix} \Sigma), S(0,0) L+L(d \Phi)_{0,0}=0\right\} .
$$

Let

$$
\begin{aligned}
\mathrm{T}_{1}(g) & =\left\{S g+(d g) \Phi: S \in \overleftrightarrow{\mathscr{E}}_{\Gamma}(\operatorname{Fix} \Sigma), \Phi \in \overrightarrow{\mathscr{E}}_{\Gamma}(\operatorname{Fix} \Sigma)\right\} \\
\overleftrightarrow{\mathscr{M}} & =\left\{S \in \overleftrightarrow{\mathscr{E}}_{\Gamma}(\operatorname{Fix} \Sigma): S(0,0)=0\right\} \\
\overrightarrow{\mathscr{M}}^{2} & =\left\{\Phi \in \overrightarrow{\mathscr{E}}_{\Gamma}(\operatorname{Fix} \Sigma): \Phi(0,0)=(d \Phi)_{0,0}=0\right\}
\end{aligned}
$$

Then $\overleftrightarrow{\mathscr{M}} g+(d g) \overrightarrow{\mathscr{M}}^{2} \subseteq \mathrm{RT}(g)$. Since

$$
\frac{\mathrm{T}_{1}(g)}{\mathrm{RT}(g)} \subseteq \frac{\mathrm{T}_{1}(g)}{\overleftrightarrow{\mathscr{M}} g+(d g) \overrightarrow{\mathscr{M}}^{2}} \quad \text { and } \quad \operatorname{dim} \frac{\mathrm{T}_{1}(g)}{\overleftrightarrow{\mathscr{M}} g+(d g) \overrightarrow{\mathscr{M}}^{2}}<\infty
$$

it follows that

$$
\mathrm{T}(g)=\mathrm{RT}(g)+\mathscr{E}_{\lambda} \cdot g_{\lambda}+\mathscr{W}
$$

with $\mathscr{W}$ a finite-dimensional vector space in $\overrightarrow{\mathscr{E}}_{\Gamma}($ Fix $\Sigma)$.

Lemma 3.8. Let $g \in \overrightarrow{\mathscr{E}}_{\Gamma}(\mathrm{Fix} \Sigma)$. Then $\mathrm{RT}(g)$ has finite codimension in $\overrightarrow{\mathscr{E}}_{\Gamma}($ Fix $\Sigma)$ if and only if $T(\mathrm{~g})$ has finite codimension.

Proof. With $\mathrm{T}_{1}(g)$ as in (3.2.14), equation (3.2.15) implies that

$$
\mathrm{T}_{1}(g)=\mathrm{RT}(g)+\mathscr{W} .
$$

By Proposition XV.2.3 of [13], $\operatorname{cod} \mathrm{T}_{1}(g)<\infty$ if and only if $\operatorname{cod} \mathrm{T}(g)<\infty$. Since $\operatorname{dim} \mathscr{W}<\infty$, it also follows that

$$
\operatorname{cod} \mathrm{RT}(g)<\infty \Longleftrightarrow \operatorname{codT}(g)<\infty .
$$

Corollary 3.9. Let $g \in \overrightarrow{\mathscr{E}}_{\Gamma}(\mathrm{Fix} \Sigma)$. Then $\mathscr{T}(g)$ has finite codimension in $\overrightarrow{\mathscr{E}}_{\Gamma}($ Fix $\Sigma)$ if and only if $T(g)$ has finite codimension.

Proof. This follows directly from the inclusions $\mathrm{RT}(g) \subseteq \mathscr{T}(g) \subseteq \mathrm{T}(g)$ and Lemma 3.8.

REMARK 3.10. The notion of geometric subgroup introduced by Damon [6] to formulate a general context for singularity theory requires four properties: naturality, tangent space structure, exponential map and filtration condition. He proves results on versality and finite determinacy for a large class of equivalence relations on map germs that satisfy these properties. It is easy to see that $\mathscr{K}_{L}$ is a geometric subgroup: in fact the proof is a simple consequence of the corresponding result for the standard equivariant theory, which is well known. For clarity we present here one part of that proof, relating to the only condition of the four above whose proof involves the linearization $L$. This part of the argument addresses the tangent space structure, and it requires the following result: for any 
$f \in \mathscr{E}_{\Gamma}($ Fix $\Sigma)$ such that $f(0,0)=0$, any $\Theta \in \mathscr{M}_{\lambda}$ and any $(S, \Phi, \Lambda) \in \mathrm{T}\left(\mathscr{K}_{L}\right)$, we must have

$$
(f S, f \Phi, \Theta \Lambda) \in \mathscr{T}\left(\mathscr{K}_{L}\right) .
$$

In fact, since $\Theta(0)=0$ then $(\Theta \cdot \Lambda)(0)=\Theta(0) \Lambda(0)=0$. Also, since Fix $\Gamma=\{0\}$, then $\Phi(0,0)=0$. Therefore, $f(0,0) S(0,0) L+L(d(f \Phi))_{0,0}=0$.

We finish this subsection with a lemma that follows from Damon's determinacy theorem [6, Theorem 10.2]. We denote by $\overrightarrow{\mathscr{M}}^{k}$ the submodule of $\overrightarrow{\mathscr{E}}_{\Gamma}($ Fix $\Sigma)$ of all mappings whose derivatives of order less than $k$ vanish at the origin.

LEMMA 3.11. If $g$ is a finite-codimension bifurcation problem then $g$ is finitely determined. That is, there exists $k>0$ such that if $p \in \overrightarrow{\mathscr{M}}^{k}$ then $g+p \sim_{\mathscr{K}_{L}} g$.

Proof. This follows immediately from Theorem 10.2 of [6] and from Corollary 3.9.

\subsection{The recognition problem and higher-order terms}

In order to solve the recognition problem for a bifurcation problem in $\overrightarrow{\mathscr{E}}_{\Gamma}($ Fix $\Sigma)$ under the group of equivalences $\mathscr{K}_{L}$ defined in (3.1.5), we require necessary and sufficient conditions for a germ to be $L$-contact equivalent to a given normal form. Here we use techniques developed by Bruce et al. [4] and Gaffney [10] to get information about orbits of group actions by studying tangent spaces to those orbits. We assume throughout that these tangent spaces are of finite codimension in $\overrightarrow{\mathscr{E}}_{\Gamma}($ Fix $\Sigma)$, so we deal with finite-codimension bifurcation problems. As mentioned in the previous section, Lemma 3.11 implies that a finite-codimension bifurcation problem is equivalent to its Taylor polynomial of order $k$ for some $k$. The recognition problem therefore reduces to the explicit characterisation of germs in a given orbit in terms of their derivatives at the origin. In consequence, it is useful to estimate higher-order terms, which are those terms that can be removed from the power series of a germ without changing its orbit. This technique provides an algebraic way to recognise those higher-order terms.

We implement the technique by way of the following subgroup of $\mathscr{K}_{L}$ :

$$
\mathscr{U}=\left\{(S, \Phi, \Lambda) \in \mathscr{K}_{L}: S(0,0)=(d \Phi)_{0,0}=I, \Lambda^{\prime}(0)=1\right\} .
$$

Here $I$ represents the $m \times m$ identity matrix. Since we assume that bifurcation problems are of finite codimension, Lemma 3.11 implies that they are finitely determined. Therefore, $\mathscr{U}$ can be considered as an algebraic group acting algebraically [20, p. 112]. Moreover, $\mathscr{U}$ is a unipotent group. We use these properties to state Lemma 3.13 below.

For a given $f \in \overrightarrow{\mathscr{E}}_{\Gamma}(\operatorname{Fix} \Sigma)$, define the tangent space to the orbit $\mathscr{U} \cdot f$ at the identity 1 (see (3.2.3)) to be the set of elements

$$
\left.\frac{d}{d t}\left(\delta_{t} \cdot f\right)\right|_{t=0}, \quad \delta_{t} \in \mathscr{U}, \quad \delta_{0}=\mathbf{1} .
$$

This set is denoted by $\mathrm{T}(f, \mathscr{U})$ and is the unipotent tangent space of $f$. A straightforward calculation yields

$$
\begin{aligned}
\mathrm{T}(f, \mathscr{U})=\{S f+(d f) \Phi: & S \in \overleftrightarrow{\mathscr{E}}_{\Gamma}(\mathrm{Fix} \Sigma), \Phi \in \overrightarrow{\mathscr{E}}_{\Gamma}(\operatorname{Fix} \Sigma), \\
& \left.S(0,0)=(d \Phi)_{0,0}=0\right\}+\mathscr{M}_{\lambda}^{2} \cdot f_{\lambda} .
\end{aligned}
$$


The restricted unipotent tangent space of $f$ is

$$
\begin{gathered}
\mathrm{RT}(f, \mathscr{U})=\{S f+(d f) \Phi: \\
S \in \overleftrightarrow{\mathscr{E}}_{\Gamma}(\operatorname{Fix} \Sigma), \Phi \in \overrightarrow{\mathscr{E}}_{\Gamma}(\operatorname{Fix} \Sigma), \\
\left.S(0,0)=(d \Phi)_{0,0}=0\right\}
\end{gathered}
$$

We also consider the following sets:

$$
\begin{gathered}
\mathscr{H}\left(f, \mathscr{K}_{L}\right)=\left\{p \in \overrightarrow{\mathscr{E}}_{\Gamma}(\operatorname{Fix} \Sigma): f+p \sim_{\mathscr{K}_{L}} f\right\}, \\
\mathscr{H}(f, \mathscr{U})=\left\{p \in \overrightarrow{\mathscr{E}}_{\Gamma}(\operatorname{Fix} \Sigma): f+p \sim_{\mathscr{U}} f\right\} .
\end{gathered}
$$

We solve the recognition problem for $f$ if we describe $\mathscr{H}\left(f, \mathscr{K}_{L}\right)$, since

$$
h \in \mathscr{K}_{L} \cdot f \quad \Longleftrightarrow \quad h-f \in \mathscr{H}\left(f, \mathscr{K}_{L}\right) .
$$

Our main objective is to describe the set of higher-order terms, which turns out to be a submodule of $\overrightarrow{\mathscr{E}}_{\Gamma}($ Fix $\Sigma)$ contained in $\mathscr{H}\left(f, \mathscr{K}_{L}\right)$ and invariant under the action of $\mathscr{K}_{L}$. We start with a definition.

Definition 3.12. A subspace of $\overrightarrow{\mathscr{E}}_{\Gamma}(\operatorname{Fix} \Sigma)$ is $\mathscr{K}_{L}$-intrinsic (or $\mathscr{U}$-intrinsic) if it is invariant under the action of $\mathscr{K}_{L}($ or $\mathscr{U})$. If a subset $\mathscr{S}$ in $\overrightarrow{\mathscr{E}}_{\Gamma}($ Fix $\Sigma)$ contains a unique maximal $\mathscr{K}_{L}$-intrinsic (or $\mathscr{U}$-intrinsic) subspace then this subspace is called the $\mathscr{K}_{L}$-intrinsic part (or the $\mathscr{U}$-intrinsic part) of $\mathscr{S}$ and is denoted $\operatorname{Itr}_{\mathscr{K}_{L}} \mathscr{S}$ (or $\operatorname{Itr}_{\mathscr{U}} S$ ).

As pointed out by Melbourne [19] a $\mathscr{U}$-intrinsic subspace is automatically a submodule, and the same argument shows that this also holds for a $\mathscr{K}_{L}$-intrinsic subspace. Melbourne also proves that if $f$ has finite codimension then

$$
\operatorname{Itr}_{\mathscr{U}} \mathscr{H}(f, \mathscr{U})=\left\{p: g+p \sim_{\mathscr{U}} f, \forall g \sim_{\mathscr{U}} f\right\} .
$$

The following lemma is a result from algebraic geometry. From Lemma 3.11 we can work modulo $\overrightarrow{\mathscr{M}}^{k}$ and so regard the unipotent group $\mathscr{U}$ as an algebraic group acting algebraically [19]. Hence, we can restate Corollary 3.5 of [4] as in [19, Corollary 3.6b].

Lemma 3.13. Suppose $f \in \overrightarrow{\mathscr{E}}_{\Gamma}(\mathrm{Fix} \Sigma)$ of finite codimension and $\mathscr{S} \subseteq \overrightarrow{\mathscr{E}}_{\Gamma}($ Fix $\Sigma)$ a $\mathscr{U}$-intrinsic submodule. Then $\mathscr{S} \subset \mathscr{H}(f, \mathscr{U})$ if and only if $\mathscr{S} \subset \mathrm{T}(f, \mathscr{U})$.

An immediate consequence is the following.

LEMMA 3.14. If $f$ has finite codimension then

$$
\operatorname{Itr}_{\mathscr{U}} \mathscr{H}(f, \mathscr{U})=\operatorname{Itr}_{\mathscr{U}} \mathrm{T}(f, \mathscr{U}) .
$$

Definition 3.15. Let $f \in \overrightarrow{\mathscr{E}}_{\Gamma}($ Fix $\Sigma)$. Define the set of higher-order terms with respect to $f$ to be the subset of $\overrightarrow{\mathscr{E}}_{\Gamma}($ Fix $\Sigma)$ given by

$$
\mathscr{P}(f)=\left\{p \in \overrightarrow{\mathscr{E}}_{\Gamma}(\text { Fix } \Sigma): g \pm p \sim_{\mathscr{K}_{L}} f, \forall g \sim_{\mathscr{K}_{L}} f\right\} .
$$

REMARK 3.16. It is obvious that $\mathscr{P}(f) \subseteq \mathscr{H}\left(f, \mathscr{K}_{L}\right)$. Moreover, $\mathscr{P}(f)$ is a $\mathscr{K}_{L^{-}}$ intrinsic submodule of $\overrightarrow{\mathscr{E}}_{\Gamma}($ Fix $\Sigma)$, the proof being the same as that of Proposition XIV.7.5 of $[\mathbf{1 3}]$. 
PROPOSITION 3.17. $\mathscr{P}(f)=\operatorname{Itr}_{\mathscr{K}_{L}} \mathscr{H}\left(f, \mathscr{K}_{L}\right)$.

Proof. From the remark above, it remains to prove that $\mathscr{P}(f)$ is unique maximal, but this follows easily using the definition of a $\mathscr{K}_{L}$-intrinsic subspace.

Next we list the inclusions between these submodules:

$$
\operatorname{Itr}_{\mathscr{K}_{L}} \mathrm{~T}(f, \mathscr{U}) \subseteq \operatorname{Itr}_{\mathscr{U}} \mathrm{T}(f, \mathscr{U})=\operatorname{Itr}_{\mathscr{U}} \mathscr{H}(f, \mathscr{U}) \subseteq \mathscr{H}\left(f, \mathscr{K}_{L}\right)
$$

The first inclusion follows because $\operatorname{Itr}_{\mathscr{K}_{L}} \mathrm{~T}(f, \mathscr{U})$ is $\mathscr{U}$-intrinsic (since $\mathscr{U} \subseteq \mathscr{K}_{L}$ ). The equality comes from Lemma 3.14. The final inclusion is obvious. Proposition 3.17 now implies that

$$
\operatorname{Itr}_{\mathscr{K}_{L}} \mathrm{~T}(f, \mathscr{U}) \subseteq \mathscr{P}(f)
$$

This inclusion is used systematically when finding higher-order terms for a normal form $f$.

\subsection{Preparation theorem for fixed-point subspaces}

Let $\Gamma$ be a compact Lie group acting linearly on the finite-dimensional spaces $V$ and $W$ and let $\Sigma$ be a subgroup of $\Gamma$. Omit the subscript $\Gamma$ in the rings to simplify notation. That is, $\mathscr{E}\left(\operatorname{Fix}_{V} \Sigma\right)$ and $\mathscr{E}\left(\operatorname{Fix}_{W} \Sigma\right)$ now denote the rings of germs of functions defined on Fix $_{V} \Sigma \times \mathbb{R}$ and Fix $_{W} \Sigma \times \mathbb{R}$ that are extendible to $\Gamma$-invariant germs defined on $V \times \mathbb{R}$ and $W \times \mathbb{R}$ respectively. Let $\varphi:\left(\operatorname{Fix}_{V} \Sigma \times \mathbb{R}, 0\right) \rightarrow\left(\operatorname{Fix}_{W} \Sigma \times \mathbb{R}, 0\right)$ be a germ extendible to a $\Gamma$-equivariant germ $\widetilde{\varphi}:(V \times \mathbb{R}, 0) \rightarrow(W \times \mathbb{R}, 0)$.

Suppose that $N$ is an $\mathscr{E}\left(\operatorname{Fix}_{V} \Sigma\right)$-module. By using the pullback $\varphi^{*}: \mathscr{E}\left(\operatorname{Fix}_{W} \Sigma\right) \rightarrow$ $\mathscr{E}\left(\operatorname{Fix}_{V} \Sigma\right)$ we can view $N$ as an $\mathscr{E}\left(\operatorname{Fix}_{W} \Sigma\right)$-module by defining, for any $f \in \mathscr{E}\left(\operatorname{Fix}_{W} \Sigma\right)$ and $n \in N$,

$$
f \cdot n:=\varphi^{*}(f) \cdot n .
$$

This is a module action since $\varphi^{*}$ is a ring homomorphism. Let $\mathscr{M}_{w}$ denote the maximal ideal in $\mathscr{E}\left(\operatorname{Fix}_{W} \Sigma\right)$, comprising all germs that vanish at the origin. If $N$ is finitely generated as an $\mathscr{E}\left(\operatorname{Fix}_{V} \Sigma\right)$-module, then the following theorem gives a necessary and sufficient condition for $N$ to be finitely generated as an $\mathscr{E}\left(\operatorname{Fix}_{W} \Sigma\right)$-module.

Theorem 3.18 (Preparation Theorem for Fixed-Point Subspaces). Let $N$ be a finitely generated $\mathscr{E}\left(\operatorname{Fix}_{V} \Sigma\right)$-module. Then, via $\varphi^{*}, N$ is a finitely generated $\mathscr{E}\left(\operatorname{Fix}_{W} \Sigma\right)$-module if and only if

$$
\operatorname{dim}\left(N / \mathscr{M}_{w} N\right)<\infty .
$$

Proof. This follows directly along the same lines as Theorem XV.8.1 of [13], using extensively the $\Gamma$-equivariant extension property of germs defined on fixed-point subspaces.

Corollary 3.19. Let $N$ be a finitely generated $\mathscr{E}\left(\operatorname{Fix}_{V} \Sigma\right)$-module. Then, via $\varphi^{*}, n_{1}, \ldots, n_{t}$ generate $N$ as an $\mathscr{E}\left(\operatorname{Fix}_{W} \Sigma\right)$-module if and only if

$$
N=\mathscr{M}_{w} N+\mathbb{R}\left\{n_{1}, \ldots, n_{t}\right\} .
$$

Proof. This is a direct consequence of Theorem 3.18 and Nakayama's Lemma. 
We can now state the main result that we need in the next subsection. Let us denote by $\mathscr{E}_{\delta}$ the ring of germs at the origin of functions $f: \mathbb{R}^{s} \rightarrow \mathbb{R}$ in $\delta$ coordinates and by $\mathscr{M}_{\delta}$ its maximal ideal of germs that vanish at the origin. Recall that, for $\delta \in \mathbb{R}^{s}$, for some $s, \mathscr{E}_{\Gamma}^{\delta}(\operatorname{Fix} \Sigma)$ denotes the ring of $s$-parameter families of germs in $\mathscr{E}_{\Gamma}($ Fix $\Sigma)$.

Lemma 3.20. For $\delta \in \mathbb{R}^{s}$, for some $s$, let $N$ be a finitely generated module over $\mathscr{E}_{\Gamma}^{\delta}(\mathrm{Fix} \Sigma)$. Then the following are equivalent:

(i) $N=\mathscr{E}_{\delta}\left\{n_{1}, \ldots, n_{t}\right\}$,

(ii) $N=\mathscr{M}_{\delta} N+\mathbb{R}\left\{n_{1}, \ldots, n_{t}\right\}$.

Proof. Define $\varphi:\left(\right.$ Fix $\left.\Sigma \times \mathbb{R} \times \mathbb{R}^{s}, 0\right) \rightarrow\left(\mathbb{R}^{s}, 0\right)$ by $\varphi(x, \lambda, \delta)=\delta$. The group $\Gamma$ acts trivially on $\mathbb{R}^{s}$, so $\varphi$ can be seen as a $\Gamma$-equivariant mapping restricted to Fix $_{V \times \mathbb{R} \times \mathbb{R}^{s}} \Sigma$ (which equals Fix ${ }_{V} \Sigma \times \mathbb{R} \times \mathbb{R}^{s}$ ). By Corollary 3.19,

if and only if

$$
N=\mathscr{E}_{\delta}\left\{n_{1}, \ldots, n_{t}\right\}
$$

$$
N=\mathscr{M}_{\delta} N+\mathbb{R}\left\{n_{1}, \ldots, n_{t}\right\}
$$

\subsection{The unfolding theorem in the hidden symmetry context}

In the context of perturbed bifurcation problems with the symmetry of a compact Lie group $\Gamma$, a major result is the $\Gamma$-Equivariant Universal Unfolding Theorem [13, Theorem XV.2.1]. This theorem gives a way to find an algebraic expression for a universal unfolding of a bifurcation problem. We now prove the analogous result for bifurcation problems with hidden symmetries. Moreover, some restrictions on the linearization are allowed: we can deal with types of bifurcations more general than those possessing an identically zero linearization at the origin. We apply this result in $\S 4.4$. The proof of the unfolding theorem for fixed-point subspaces follows the same steps as the proof of the equivariant version. We shall present the complete proof, since it requires some additional facts such as uniqueness of solutions of ordinary differential equations, rather than just the existence of equivariant extensions.

Theorem 3.21 (Unfolding Theorem for Fixed-Point Subspaces). Let $g \in \overrightarrow{\mathscr{E}}_{\Gamma}($ Fix $\Sigma)$ and let $G \in \overrightarrow{\mathscr{E}}_{\Gamma}^{\alpha}($ Fix $\Sigma)$ be a k-parameter unfolding of g. Then $G$ is versal if and only if

$$
\overrightarrow{\mathscr{E}}_{\Gamma}(\operatorname{Fix} \Sigma)=\mathrm{T}(g)+\mathbb{R}\left\{G_{\alpha_{1}}(x, \lambda, 0), \ldots, G_{\alpha_{k}}(x, \lambda, 0)\right\}
$$

REMARK 3.22. In the discussion on the definition of $\mathrm{T}(g)$ given in $\S 3.2$, we have proved that (3.5.1) is a necessary condition for $G$ to be versal (see (3.2.9)). To prove this is also a sufficient condition, we need some definitions and results which we present below.

DeFinition 3.23. By analogy with the definition of $\mathrm{T}_{1}(g)$ for a germ $g \in \overrightarrow{\mathscr{E}}_{\Gamma}\left(\right.$ Fix $\Sigma$ ) (see (3.2.14)), we define the following submodule of $\overrightarrow{\mathscr{E}}_{\Gamma}^{\delta}($ Fix $\Sigma)$ for an $s$-parameter unfolding $K$ of $g$ :

$$
\mathrm{T}_{1}^{u}(K)=\left\{S K+(d K) X: S \in \overleftrightarrow{\mathscr{E}}_{\Gamma}^{\delta}(\operatorname{Fix} \Sigma), X \in \overrightarrow{\mathscr{E}}_{\Gamma}^{\delta}(\operatorname{Fix} \Sigma)\right\}
$$


We also define the extended tangent space

$$
\mathrm{T}^{u}(K)=\mathrm{T}_{1}^{u}(K)+\mathscr{E}_{\lambda, \delta} \cdot K_{\lambda}
$$

Proposition 3.24. Let $g \in \overrightarrow{\mathscr{E}}_{\Gamma}(\mathrm{Fix} \Sigma)$ be a finite-codimension germ and let $K \in \overrightarrow{\mathscr{E}}_{\Gamma}(\mathrm{Fix} \Sigma)$ be an s-parameter unfolding of $g$, where $\delta=\left(\delta_{1}, \ldots, \delta_{s}\right)$. Let $q_{1}, \ldots, q_{r} \in \overrightarrow{\mathscr{E}}_{\Gamma} \delta(\operatorname{Fix} \Sigma)$. Then the following are equivalent:

(i) $\overrightarrow{\mathscr{E}}_{\Gamma}($ Fix $\Sigma)=\mathrm{T}(g)+\mathbb{R}\left\{q_{1}(x, \lambda, 0), \ldots, q_{r}(x, \lambda, 0)\right\}$,

(ii) $\overrightarrow{\mathscr{E}}_{\Gamma}^{\delta}(\operatorname{Fix} \Sigma)=\mathrm{T}^{u}(K)+\mathscr{E}_{\delta}\left\{q_{1}(x, \lambda, \delta), \ldots, q_{r}(x, \lambda, \delta)\right\}$.

Proof. This result refers only to extended tangent spaces in $\overrightarrow{\mathscr{E}}_{\Gamma}($ Fix $\Sigma)$ and in $\overrightarrow{\mathscr{E}}_{\Gamma}^{\delta}($ Fix $\Sigma)$ whose definitions coincide with the classical definitions given for equivariant germs defined on the whole space $V$. Therefore, its proof is similar to the proof of Corollary XV.7.2 of [13]. In our case, the result corresponding to Lemma XV.7.1 of [13], used in the proof of Corollary XV.7.2 [13], is Lemma 3.20.

Definition 3.25. Germs $G$ and $H$ as in Definition 3.6 are $u$-isomorphic (isomorphic as unfoldings) if $A$ in (3.2.1) is a diffeomorphism germ.

Definition 3.26. Consider $G$ a $k$-parameter unfolding of $g$ and let $A:\left(\mathbb{R}^{l}, 0\right) \rightarrow\left(\mathbb{R}^{k}, 0\right)$. Define the pullback unfolding $A^{*} G \in \overrightarrow{\mathscr{E}}_{\Gamma}^{\beta}($ Fix $\Sigma)$ to be

$A^{*} G:\left(\right.$ Fix $\left.\Sigma \times \mathbb{R} \times \mathbb{R}^{l}, 0\right) \rightarrow$ Fix $\Sigma$,

$$
(x, \lambda, \beta) \mapsto A^{*} G(x, \lambda, \beta)=G(x, \lambda, A(\beta)) .
$$

It is obvious that $A^{*} G$ is also an unfolding of $g$, but $A^{*} G$ has $l$ unfolding parameters rather than the $k$ that $G$ has.

Now we start the proof that (3.5.1) is a sufficient condition for $G$ to be versal. Let $H(x, \lambda, \beta)$ be an $l$-parameter unfolding of $g$. We must show that $H$ factors through $G$. Consider the following unfolding of $g$ :

$$
K(x, \lambda, \alpha, \beta)=G(x, \lambda, \alpha)+H(x, \lambda, \beta)-g(x, \lambda) .
$$

Then $H$ factors through $K$ in a trivial way. We claim that there exists a submersion $A:\left(\mathbb{R}^{k} \times \mathbb{R}^{l}, 0\right) \rightarrow\left(\mathbb{R}^{k}, 0\right)$ such that $K$ is u-isomorphic to $A^{*} G$. Before proving this claim, we check that this establishes the theorem:

$$
K(x, \lambda, \alpha, \beta)=S(x, \lambda, \alpha, \beta) G(X(x, \lambda, \alpha, \beta), \Lambda(\lambda, \alpha, \beta), A \circ \phi(\alpha, \beta)),
$$

with $\phi:\left(\mathbb{R}^{k+l}, 0\right) \rightarrow\left(\mathbb{R}^{k+l}, 0\right)$ a germ of diffeomorphism. So

$$
H(x, \lambda, \beta)=S(x, \lambda, 0, \beta) G(X(x, \lambda, 0, \beta), \Lambda(\lambda, 0, \beta), A \circ \phi(0, \beta)) .
$$

Therefore, $H$ factors through $G$. So now it remains to prove that the submersion $A$ exists, which we do by induction on $l$.

For $l=0$ the claim is obviously true: just let $A$ be the identity. Assume the claim is true for $l-1$, with $\mathbb{R}^{l-1}$ embedded in $\mathbb{R}^{l}$ by $\left(\beta_{1}, \ldots, \beta_{l-1}\right) \mapsto$ $\left(\beta_{1}, \ldots, \beta_{l-1}, 0\right)$. If we define $F\left(x, \lambda, \alpha, \beta_{1}, \ldots, \beta_{l-1}\right)=K\left(x, \lambda, \alpha, \beta_{1}, \ldots, \beta_{l-1}, 0\right)$ then, by induction, there exists a submersion $B:\left(\mathbb{R}^{k} \times \mathbb{R}^{l-1}, 0\right) \rightarrow\left(\mathbb{R}^{k}, 0\right)$ such that $B^{*} G$ is u-isomorphic to $F$. It therefore suffices to find a submersion $C:\left(\mathbb{R}^{k} \times \mathbb{R}^{l}, 0\right) \rightarrow\left(\mathbb{R}^{k} \times \mathbb{R}^{l-1}, 0\right)$ such that $C^{*} F$ is u-isomorphic to $K$. For then we may set $A=B \circ \phi_{1} \circ C \circ \phi_{2}$, where $\phi_{1}:\left(\mathbb{R}^{k} \times \mathbb{R}^{l-1}, 0\right) \rightarrow\left(\mathbb{R}^{k} \times \mathbb{R}^{l-1}, 0\right)$ is 
the diffeomorphism from the u-isomorphism between $B^{*} G$ and $F$, and $\phi_{2}:\left(\mathbb{R}^{k} \times \mathbb{R}^{l}, 0\right) \rightarrow\left(\mathbb{R}^{k} \times \mathbb{R}^{l}, 0\right)$ is the diffeomorphism from the u-isomorphism between $C^{*} F$ and $K$.

To ease notation, set $\delta=\left(\delta_{1}, \ldots, \delta_{k+l}\right)$, with $\delta_{1}=\alpha_{1}, \ldots, \delta_{k}=\alpha_{k}, \delta_{k+1}=\beta_{1}$, $\ldots, \delta_{k+l}=\beta_{l}$. From (3.5.1) we have

$$
\overrightarrow{\mathscr{E}}_{\Gamma}(\operatorname{Fix} \Sigma)=\mathrm{T}(g)+\mathbb{R}\left\{K_{\delta_{1}}, \ldots, K_{\delta_{k}}\right\}_{\delta=0} .
$$

By Proposition 3.24,

$$
\overrightarrow{\mathscr{E}}_{\Gamma}^{\delta}(\operatorname{Fix} \Sigma)=\mathrm{T}^{u}(K)+\mathscr{E}_{\delta}\left\{K_{\alpha_{1}}, \ldots, K_{\alpha_{k}}\right\} .
$$

Set $s=k+l$. Since $\mathrm{T}^{u}(K)=\{S K+(d K) X\}+\mathscr{E}_{\lambda, \delta} \cdot K_{\lambda} \quad$ and $\quad-K_{\delta_{s}}=-K_{\beta_{l}} \in$ $\overrightarrow{\mathscr{E}}_{\Gamma}^{\delta}($ Fix $\Sigma)$, we have

$$
-K_{\delta_{s}}=S K+(d K) X+\Lambda(\lambda, \delta) K_{\lambda}+\sum_{i=1}^{s-1} \xi_{i}(\delta) K_{\delta_{i}},
$$

where $\xi_{i}(\delta)=0$ if $i>k$. We can write

$$
(d K) X=\sum_{j=1}^{m} X_{j} \frac{\partial K}{\partial x_{j}}
$$

where by $\partial K / \partial x_{j}$ we mean the vector $\left(\partial K_{1} / \partial x_{j}, \ldots, \partial K_{m} / \partial x_{j}\right)^{\mathrm{t}}$. So we are left with

$$
-K_{\delta_{s}}=S K+\sum_{j=1}^{m} X_{j} \frac{\partial K}{\partial x_{j}}+\Lambda(\lambda, \delta) K_{\lambda}+\sum_{i=1}^{s-1} \xi_{i}(\delta) K_{\delta_{i}} .
$$

Consider the system of ordinary differential equations

$$
\begin{aligned}
\frac{d \delta_{s}}{d t} & =1, \\
\frac{d x}{d t} & =X(x, \lambda, \delta), \\
\frac{d \lambda}{d t} & =\Lambda(\lambda, \delta), \\
\frac{d \delta_{i}}{d t} & =\xi_{i}(\delta), \quad \text { for } i=1, \ldots, s-1,
\end{aligned}
$$

and suppose that $\left(\delta_{s}(t), x(t), \lambda(t), \delta_{i}(t)\right)$ is a solution of this system. Then (3.5.2) can be written as

$$
\frac{d}{d t} K(x(t), \lambda(t), \delta(t))=-S(x(t), \lambda(t), \delta(t)) K(x(t), \lambda(t), \delta(t)) .
$$

At $t=0$, take the initial conditions to be $\delta_{s}=0, x=x_{0}, \lambda=\lambda_{0}, \delta_{i}=\delta_{i, 0}$ for $i=1, \ldots, s-1$. Since $d \delta_{s} / d t=1$, the solution curve $P(t)$ of (3.5.3) at $P(0)=\left(0, x_{0}, \lambda_{0}, \delta_{1,0}, \ldots, \delta_{m-1,0}\right)$ is transverse to the hyperplane $\left\{\delta_{s}=0\right\}$. Define the map

$$
\varphi:\left(\text { Fix } \Sigma \times \mathbb{R} \times \mathbb{R}^{s}, 0\right) \rightarrow \text { Fix } \Sigma \times \mathbb{R} \times \mathbb{R}^{s-1}
$$

by projecting $(x, \lambda, \delta)$ along the integral curves until $\delta_{s}=0$. Then $\varphi$ is smooth (see [1]) and $\left.\varphi\right|_{\delta_{s}=0}$ is the identity. We can write $\varphi$ as

$$
\varphi(x, \lambda, \delta)=(\rho(x, \lambda, \delta), \Lambda(\lambda, \delta), C(\delta)),
$$


with $\left.\rho(x, \lambda, \delta)\right|_{\delta_{s}=0}=x$ and $\left.\Lambda(\lambda, \delta)\right|_{\delta_{s}=0}=\lambda$. The Jacobian $(D \varphi)_{0}$ has the form

$$
\begin{array}{cccc}
x & \lambda & \delta_{1} \ldots \delta_{s} \\
x & (d \rho)_{0} & * & * \\
\lambda & 0 & \left(d_{\lambda} \Lambda\right)_{0} & * \\
\delta_{1} & & & \\
\vdots & 0 & 0 & \left(d_{\delta} C\right)_{0} \\
\delta_{s-1} & & &
\end{array}
$$

and therefore $\varphi$ is a submersion. Also

(i) $\operatorname{rank}\left(d_{\delta} C\right)_{0}=s-1$, so $C$ is a submersion,

(ii) $\left(d_{\lambda} \Lambda\right)_{0}$ is non-singular, so $\Lambda(\cdot, \delta)$ is a diffeomorphism for each $\delta$,

(iii) $(d \rho)_{0}$ is non-singular, so $\rho(\cdot, \lambda, \delta)$ is a diffeomorphism for each $(\lambda, \delta)$.

We now show that $\rho:\left(\right.$ Fix $\left.\Sigma \times \mathbb{R} \times \mathbb{R}^{s}, 0\right) \rightarrow$ Fix $\Sigma$ is extendible to a $\Gamma$ equivariant germ. To do so, extend the domain of the system (3.5.3) to $\mathrm{V} \times \mathbb{R} \times \mathbb{R}^{s}$. Then, the second equation becomes

$$
\frac{d \widetilde{x}}{d t}=\widetilde{X}(\widetilde{x}, \lambda, \delta),
$$

where $\widetilde{X}$ is the $\Gamma$-equivariant extension of $X$. For the extended domain we can define a germ $\psi:\left(\mathrm{V} \times \mathbb{R} \times \mathbb{R}^{s}, 0\right) \rightarrow \mathrm{V} \times \mathbb{R} \times \mathbb{R}^{s-1}$ for the new system in the same way as $\varphi$ is defined for the system (3.5.3), getting

$$
\psi(\widetilde{x}, \lambda, \delta)=(R(\widetilde{x}, \lambda, \delta), \Lambda(\lambda, \delta), C(\delta)) .
$$

As for $\rho$, we have $\left.R(\widetilde{x}, \lambda, \delta)\right|_{\delta_{s}=0}=\widetilde{x}$. We also have

$$
\begin{aligned}
\frac{d}{d t} R(\gamma \widetilde{x}, \lambda, \delta) & =\widetilde{X}(R(\gamma \widetilde{x}, \lambda, \delta), \lambda, \delta), \\
\frac{d}{d t} \gamma R(\widetilde{x}, \lambda, \delta) & =\gamma \frac{d}{d t} R(\widetilde{x}, \lambda, \delta)=\gamma \widetilde{X}(R(\widetilde{x}, \lambda, \delta), \lambda, \delta) \\
& =\widetilde{X}(\gamma R(\widetilde{x}, \lambda, \delta), \lambda, \delta), \\
\left.\gamma R(\widetilde{x}, \lambda, \delta)\right|_{t=0} & =\gamma \widetilde{x}=\left.R(\gamma \widetilde{x}, \lambda, \delta)\right|_{t=0} .
\end{aligned}
$$

By the uniqueness of solutions of ordinary differential equations, $R(\gamma \widetilde{x}, \lambda, \delta)=$ $\gamma R(\widetilde{x}, \lambda, \delta)$, for all $\gamma \in \Gamma$. Since

we have

$$
\frac{d}{d t} R(\widetilde{x}, \lambda, \delta)=\widetilde{X}(R(\widetilde{x}, \lambda, \delta))
$$

$$
\begin{aligned}
\left.\frac{d}{d t} R\right|_{\text {Fix } \Sigma \times \mathbb{R} \times \mathbb{R}^{s}}(x, \lambda, \delta) & =\widetilde{X}\left(\left.R\right|_{\text {Fix } \Sigma \times \mathbb{R} \times \mathbb{R}^{s}}(x, \lambda, \delta), \lambda, \delta\right) \\
& =X\left(\left.R\right|_{\text {Fix } \Sigma \times \mathbb{R} \times \mathbb{R}^{s}}(x, \lambda, \delta), \lambda, \delta\right) .
\end{aligned}
$$

Also, for all $x \in \operatorname{Fix} \Sigma,\left.R(x, \lambda, \delta)\right|_{t=0}=x=\left.\rho(x, \lambda, \delta)\right|_{t=0}$. Therefore, again by

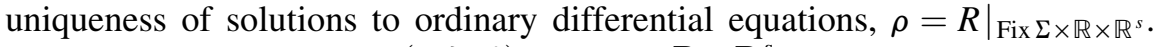

Given a point $P=(x, \lambda, \delta) \in$ Fix $\Sigma \times \mathbb{R} \times \mathbb{R}^{s}$, select the integral curve $(x(t), \lambda(t), \delta(t))$ of (3.5.3) passing through $P$. Now consider the non-autonomous 
ordinary differential equation

$$
\frac{d y}{d t}=-S(x(t), \lambda(t), \delta(t)) \cdot y,
$$

with $y \in$ Fix $\Sigma$ and $S$ from (3.5.4). Note that (3.5.8) depends only on $(x(0), \lambda(0), \delta(0))$ and the initial condition $y(0)=y_{0}$, since $(x(t), \lambda(t), \delta(t))$ is obtained by integrating (3.5.3). Therefore, the solution of (3.5.8) is of the form

$$
y(t)=Y\left(y_{0}, t, x(0), \lambda(0), \delta(0)\right)=Y\left(y_{0}, t, \varphi(x, \lambda, \delta), 0\right) .
$$

For each fixed $t$, and for $(x(0), \lambda(0), \delta(0))$ near zero, the map $y_{0} \mapsto y(t)$ is a local diffeomorphism [3, Chapter 2, Corollary 9].

Now (3.5.4) says that $K(x(t), \lambda(t), \delta(t))$ satisfies (3.5.8). So

$$
K(x(t), \lambda(t), \delta(t))=Y(K(x(0), \lambda(0), \delta(0)), t, x(0), \lambda(0), \delta(0)) .
$$

Therefore

$$
K(x, \lambda, \delta)=E_{x, \lambda, \delta}(K(\rho(x, \lambda, \delta), \Lambda(\lambda, \delta), C(\delta), 0))
$$

where

$$
E_{x, \lambda, \delta}(y)=Y\left(y, \delta_{s}, \rho(x, \lambda, \delta), \Lambda(\lambda, \delta), C(\delta), 0\right)
$$

is a family of diffeomorphisms on Fix $\Sigma$. From (3.5.10) we have

$$
\begin{aligned}
K(x, \lambda, \delta) & =E_{x, \lambda, \delta}(F(\rho(x, \lambda, \delta), \Lambda(\lambda, \delta), C(\delta)) \\
& =E_{x, \lambda, \delta}\left(C^{*} F(\rho(x, \lambda, \delta), \Lambda(\lambda, \delta), \delta)\right) .
\end{aligned}
$$

Finally, we prove that there exists an invertible $T \in \overleftrightarrow{\mathscr{E}}_{\Gamma}^{\delta}($ Fix $\Sigma)$ such that

$$
E_{x, \lambda, \delta}\left(C^{*} F(\rho(x, \lambda, \delta), \Lambda(\lambda, \delta), \delta)\right)=T(x, \lambda, \delta) \cdot C^{*} F(\rho(x, \lambda, \delta), \Lambda(\lambda, \delta), \delta) .
$$

To do so, we extend the ordinary differential equation (3.5.8) in two ways:

$$
\begin{aligned}
& \text { (a) } \frac{d \widetilde{y}}{d t}=-\widetilde{S}(\widetilde{x}(t), \lambda(t), \delta(t)) \cdot \widetilde{y}, \\
& \text { (b) } \frac{d \widetilde{y}}{d t}=-\widetilde{S}(x(t), \lambda(t), \delta(t)) \cdot \widetilde{y},
\end{aligned}
$$

where $\left.\widetilde{y}\right|_{\text {Fix } \Sigma}=y,\left.\widetilde{x}\right|_{\text {Fix } \Sigma}=x$ and $\widetilde{S}$ is an extension of $S$ to $\mathrm{V} \times \mathbb{R} \times \mathbb{R}^{s}$. So in the same way as we have $E_{x, \lambda, \delta}$ for (3.5.8), we also have the corresponding diffeomorphisms

$$
\begin{aligned}
& \text { (a) } E_{\tilde{x}, \lambda, \delta}^{1}(\widetilde{y})=\widetilde{Y}^{1}\left(\widetilde{y}, \delta_{s}, \psi(\widetilde{x}, \lambda, \delta), 0\right), \\
& \text { (b) } E_{x, \lambda, \delta}^{2}(\widetilde{y})=\widetilde{Y}^{2}\left(\widetilde{y}, \delta_{s}, \varphi(x, \lambda, \delta), 0\right),
\end{aligned}
$$

for (3.5.11)(a) and (3.5.11)(b) respectively. Since

$$
\begin{aligned}
E_{x, \lambda, \delta}^{1}(\widetilde{y})=\widetilde{Y}^{1}\left(\widetilde{y}, \delta_{s}, \psi(x, \lambda, \delta), 0\right) & =\widetilde{Y}^{2}\left(\widetilde{y}, \delta_{m}, \varphi(x, \lambda, \delta), 0\right) \\
& =E_{x, \lambda, \delta}^{2}(\widetilde{y}),
\end{aligned}
$$

we get

$$
\begin{aligned}
K(x, \lambda, \delta)=E_{x, \lambda, \delta}(K(\varphi(x, \lambda, \delta), 0)) & =E_{x, \lambda, \delta}^{2}(K(\varphi(x, \lambda, \delta), 0)) \\
& =E_{x, \lambda, \delta}^{1}(K(\varphi(x, \lambda, \delta), 0)),
\end{aligned}
$$


where the second equality follows from uniqueness of solutions of (3.5.11)(b) for a given initial condition.

Next we verify two properties (P1) and (P2) of $E^{1}$, as follows.

(P1) $E_{\gamma \tilde{x}, \lambda, \delta}^{1}(\gamma \widetilde{y})=\gamma E_{\tilde{x}, \lambda, \delta}^{1}(\widetilde{y})$. In fact, for the integral curve $\widetilde{y}(t)=$ $\widetilde{Y}^{1}(\widetilde{y}, t, \widetilde{x}(0), \lambda(0), \delta(0))$, consider the curve defined by

Now

$$
\gamma \cdot \widetilde{y}(t)=\gamma \cdot \widetilde{Y}^{1}(\widetilde{y}, t, \widetilde{x}(0), \lambda(0), \delta(0)) .
$$

$$
\frac{d \gamma \widetilde{y}}{d t}=\gamma \frac{d \widetilde{y}}{d t}=\gamma(-\widetilde{S}(\widetilde{x}(t), \lambda(t), \delta(t))) \widetilde{y}=-\widetilde{S}(\gamma \widetilde{x}(t), \lambda(t), \delta(t)) \gamma \widetilde{y}
$$

and

$$
(\gamma \widetilde{y})(0)=\gamma \widetilde{y}_{0} .
$$

Therefore, $\gamma \cdot \widetilde{Y}^{1}\left(\widetilde{y}_{0}, \delta_{s}, \widetilde{x}(0), \lambda(0), \delta(0)\right)=\widetilde{Y}^{1}\left(\gamma \widetilde{y}_{0}, \delta_{s}, \gamma \widetilde{x}(0), \lambda(0), \delta(0)\right)$. That is,

$$
E_{\gamma \tilde{x}, \lambda, \delta}^{1}(\gamma \widetilde{y})=\gamma E_{\tilde{x}, \lambda, \delta}^{1}(\widetilde{y}) .
$$

(P2) $E_{\tilde{x}, \lambda, \delta}^{1}(0)=0$. In fact,

$$
E_{\tilde{x}, \lambda, \delta}^{1}(0)=\widetilde{Y}\left(0, \delta_{s}, \widetilde{x}(0), \lambda(0), \delta(0)\right)=\widetilde{Y}(0, t=0, \widetilde{x}(0), \lambda(0), \delta(0))=0 .
$$

This completes the verification of (P1) and (P2).

Since the diffeomorphism $E^{1}$ satisfies (P1) and (P2), Proposition 3.1 implies that there exists a matrix germ $\widetilde{T}$ satisfying

$$
\widetilde{T}(\gamma \widetilde{x}, \lambda, \delta) \gamma=\gamma \widetilde{T}(\widetilde{x}, \lambda, \delta)
$$

with $\widetilde{T}(0,0,0)$ invertible, such that

$$
E_{x, \lambda, \delta}^{1}(K(\varphi(x, \lambda, \delta), 0))=\widetilde{T}(x, \lambda, \delta) \cdot K(\varphi(x, \lambda, \delta), 0) .
$$

Finally, define

$$
T=\left.\widetilde{T}\right|_{\text {Fix } \Sigma \times \mathbb{R} \times \mathbb{R}^{s}}
$$

to get $T \in \overleftrightarrow{\mathscr{E}}_{\Gamma}^{\delta}(\operatorname{Fix} \Sigma)$, where $T(0,0,0)$ is invertible and (3.5.13) becomes

$$
K(x, \lambda, \delta)=T(x, \lambda, \delta) \cdot C^{*} F(\rho(x, \lambda, \delta), \Lambda(\lambda, \delta), \delta) .
$$

Therefore, $K$ is u-isomorphic to $C^{*} F$ as required.

\section{Steady states of partial differential equations on generalized rectangles: singularity theory}

We now apply the theory developed so far to a particular class of singularities with hidden symmetries, those arising by Liapunov-Schmidt reduction in connections with the steady state bifurcation of certain partial differential equations defined on generalized (that is, multidimensional) rectangles.

Consider the partial differential equation

$$
u_{t}+F(u, \lambda)=0 .
$$

Here $u$ is defined on an $N$-dimensional rectangle $\mathrm{R}=\left[0, \pi L_{1}\right] \times \ldots \times$ $\left[0, \pi L_{N}\right] \subset \mathbb{R}^{N}$ with the $L_{j}$ all distinct, and $F$ is an elliptic operator equivariant under the group $\mathbf{O}(2)^{N}$ generated by coordinate reflections and translations modulo a periodic lattice, that is, modulo $2 \pi L_{j}$ along the direction $x_{j}$ for 
$1 \leqslant j \leqslant N$. Let $\lambda$ be a distinguished parameter such that $F(0, \lambda) \equiv 0$. Assume (4.0.1) holds under Neumann boundary conditions

$$
\frac{\partial}{\partial x_{j}} u(x)=0 \quad \text { for } x_{j}=0, \pi L_{j} \text { with } 1 \leqslant j \leqslant N .
$$

To simplify the notation in (4.0.2) we omit the variable $t$ from $u$, since we are interested in steady-state solutions.

REMARK 4.1. As discussed in $\S 1$, steady-state bifurcations of (4.0.1) can be projected into $\operatorname{ker}(d F)_{0,0}$. It is well known [13] that the presence of symmetries of a compact Lie group $\Gamma$ forces 0 -eigenvalues of high multiplicity, so we may expect to find bifurcation problems where $\operatorname{ker}(d F)_{0,0}$ has dimension greater than 1. When the entire $\operatorname{ker}(d F)_{0,0}$ is $\Gamma$-irreducible, that is, the only $\Gamma$-invariant subspaces of $\operatorname{ker}(d F)_{0,0}$ are the trivial subspaces, then we have a single-mode bifurcation problem. When $\operatorname{ker}(d F)_{0,0}$ decomposes as a direct sum of two $\Gamma$ irreducible subspaces $V_{1} \oplus V_{2}$, we have a two-mode interaction.

Gomes and Stewart [14] applied the technique of domain extension developed by Field et al. [8] to prove that a two-mode interaction for (4.0.1) satisfying Neumann boundary conditions corresponds to a bifurcation problem defined on $\mathbb{C}^{2^{N}}$ whose symmetry group is $\mathbf{O}(2)^{N}$, on the restricted domain given by the subspace Fix $\mathbf{Z}_{2}^{N}$. Their method is to decompose $\mathbb{C}^{2^{N}}$ as $\mathbb{C}^{2^{N-1}} \times \mathbb{C}^{2^{N-1}}$ and define an irreducible action of $\mathbf{O}(2)^{N}$ on each component as follows. Denote the first component by $V_{\mathbf{k}}=\mathbb{C}^{2^{N-1}}$ and the second component by $V_{\boldsymbol{\ell}}=\mathbb{C}^{2^{N-1}}$. Define mode vectors $\mathbf{k}=\left(k_{1}, \ldots, k_{N}\right)$ and $\ell=\left(\ell_{1}, \ldots, \ell_{N}\right)$ with $k_{j}$ and $\ell_{j}$ non-zero and positive, for $j=1, \ldots, N$. Then:

(i) for $\theta=\left(\theta_{1}, \ldots, \theta_{N}\right) \in \mathbf{T}^{N}$ the action on each direction $z_{j}$, for $1 \leqslant j \leqslant 2^{N}$, is defined by

$$
\theta \cdot z_{j}=e^{i \varepsilon_{j} \cdot \theta} z_{j}
$$

where the $\varepsilon_{j}$ are all the elements of the form $\left(a_{1} / L_{1}, \pm a_{2} / L_{2}, \ldots, \pm a_{N} / L_{N}\right)$, with $a_{j}=k_{j}$ for the first $2^{N-1}$ directions and $a_{j}=\ell_{j}$ for the remainder;

(ii) $\mathbf{Z}_{2}^{N}$ acts on each component $\mathbb{C}^{2^{N-1}}$ by

(a) the flip $\left(z_{1}, z_{2}, \ldots, z_{2^{N-1}}\right) \mapsto\left(\bar{z}_{2^{N-1}}, \ldots, \bar{z}_{2}, \bar{z}_{1}\right)$,

(b) all the other $N-1$ generators act by permutation.

The entries in the mode vectors depend on the original problem (4.0.1), but a simple calculation using (4.0.3) implies, as Armbruster and Dangelmayr [2] concluded for $N=1$, that we may factor out the kernel of the action to assume that $k_{j}$ and $\ell_{j}$ are coprime for $j=1, \ldots, N$.

\subsection{The equations}

Clearly Fix $\mathbf{Z}_{2}^{N}$ is the subspace of $\mathbb{C}^{2^{N}}$ isomorphic to $\mathbb{R}^{2}$ comprising all elements

$$
(\underbrace{x, \ldots, x}_{2^{N-1}}, \underbrace{y, \ldots, y}_{2^{N-1}}) .
$$

In [14] Gomes and Stewart state that generators of $\mathscr{E}_{\mathbf{O}(2)^{2}}$ have to be computed 
algorithmically, since no simple general formula covers all cases. They also explain that following the same method for $\mathscr{E}_{\mathbf{O}(2)^{N}}$ leads to a very complicated problem. So they present a different approach to give a minimal set of generators for $\mathscr{E}_{\mathbf{O}(2)^{N}}\left(\right.$ Fix $\left.\mathbf{Z}_{2}^{N}\right)$ by direct computation on Fix $\mathbf{Z}_{2}^{N}$, instead of finding generators for $\mathscr{E}_{\mathbf{O}(2)^{N}}$ and then restricting those to Fix $\mathbf{Z}_{2}^{N}$. The result is the following.

THEOREM 4.2. Let the group $\mathbf{O}(2)^{N}$ act on $V_{\mathbf{k}} \oplus V_{\mathbb{\ell}}$ as in (4.0.3) and (4.0.4). Then the $\mathbf{O}(2)^{N}$-invariants restricted to Fix $\mathbf{Z}_{2}^{N}$ are generated as follows.

Case 1. If all $k_{j}$ have the same parity and all $\ell_{j}$ have the same parity (not necessarily the same as the $k_{j}$ ), then the generators are $u=x^{2}, v=y^{2}$ and $w=x^{n} y^{m}$, where $m=\max _{j} k_{j}$ and $n=\max _{j} \ell_{j}$.

Case 2. Otherwise, the generators are $u=x^{2}$ and $v=y^{2}$.

Proof. See [14, Theorem 6].

Gomes and Stewart also prove [14, Theorem 5] that the equivariants restricted to Fix $\mathbf{Z}_{2}^{N}$ are generated over $\mathscr{E}_{\mathbf{O}(2)^{N}}\left(\right.$ Fix $\left.\mathbf{Z}_{2}^{N}\right)$ as follows.

For Case 1, the generators are

$$
g_{1}=\left(\begin{array}{c}
x \\
\vdots \\
x \\
0 \\
\vdots \\
0
\end{array}\right), \quad g_{2}=\left(\begin{array}{c}
0 \\
\vdots \\
0 \\
y \\
\vdots \\
y
\end{array}\right), \quad g_{3}=\left(\begin{array}{c}
x^{n-1} y^{m} \\
\vdots \\
x^{n-1} y^{m} \\
0 \\
\vdots \\
0
\end{array}\right), \quad g_{4}=\left(\begin{array}{c}
0 \\
\vdots \\
0 \\
x^{n} y^{m-1} \\
\vdots \\
x^{n} y^{m-1}
\end{array}\right) \text {, }
$$

where $m=\max _{j} k_{j}$ and $n=\max _{j} \ell_{j}$.

For Case 2, the generators are $g_{1}$ and $g_{2}$ of (4.1.1).

In Case 1 , any $g \in \overrightarrow{\mathscr{E}}_{\mathbf{O}(2)^{N}}\left(\operatorname{Fix} \mathbf{Z}_{2}^{N}\right)$ is of the form

$$
g(x, \ldots, x, y, \ldots, y, \lambda)=\left(\begin{array}{c}
a(u, v, \lambda) x+b(u, v, \lambda) x^{n-1} y^{m} \\
\vdots \\
a(u, v, \lambda) x+b(u, v, \lambda) x^{n-1} y^{m} \\
c(u, v, \lambda) y+d(u, v, \lambda) x^{n} y^{m-1} \\
\vdots \\
c(u, v, \lambda) y+d(u, v, \lambda) x^{n} y^{m-1}
\end{array}\right),
$$

where $u=x^{2}$ and $v=y^{2}$. Here the invariant $w=x^{n} y^{m}$ is not required to write (4.1.2). For Case 2, we have (4.1.2) taking $b \equiv d \equiv 0$. Then, we can consider $\overrightarrow{\mathscr{E}}_{\mathbf{O}(2)^{N}}\left(\right.$ Fix $\left.\mathbf{Z}_{2}^{N}\right)$ as a module over the ring generated by $u, v$ and $\lambda$, and omit $w$ in the first case. So for both cases we may consider $\mathscr{E}_{\mathbf{O}(2)^{N}}\left(\operatorname{Fix} \mathbf{Z}_{2}^{N}\right)$ to be generated by $u, v$ and $\lambda$.

The bifurcation equations are then

$$
\left\{\begin{array}{l}
a(u, v, \lambda) x+b(u, v, \lambda) x^{n-1} y^{m}=0, \\
c(u, v, \lambda) y+d(u, v, \lambda) x^{n} y^{m-1}=0,
\end{array}\right.
$$


for the first case, and

$$
\left\{\begin{array}{l}
a(u, v, \lambda) x=0 \\
c(u, v, \lambda) y=0
\end{array}\right.
$$

for the second case.

The system (4.1.4) does not occur when $N=1$. It represents the zero set of a one-parameter germ on $\mathbb{R}^{2}$ equivariant under the standard action of the group $\mathbf{Z}_{2} \oplus \mathbf{Z}_{2}$. Golubitsky and Schaeffer [12] give the normal form for the least degenerate bifurcation (with topological codimension 1, namely $\mathbf{Z}_{2} \oplus \mathbf{Z}_{2}$ codimension 3 and modality 2). In [17] Manoel presents a classification of such bifurcations up to topological codimension 2 , namely $\mathbf{Z}_{2} \oplus \mathbf{Z}_{2}$-codimension 3 and modality 1 . The system (4.1.3) includes the same equations as for $N=1$, but it is not immediately evident that the same singularity-theoretic equivalence relation is appropriate when $N>1$. When $N=1$, Armbruster and Dangelmayr [2] classified such problems up to topological codimension 2, assuming $m$ and $n$ to be coprime. However, when $N>1$, the numbers $m$ and $n$ can no longer be assumed coprime. For example, for $N=4$ the modes could be $\mathbf{k}=(2,6,4,2)$ and $\ell=(3,1,9,1)$, in which case $m=6$ and $n=9$ with common factor 3 . In $\S 4.3$ we pursue the implications of this possibility by considering the case $m=n=3$.

In $\S 4.4$ we study the zeros of (4.1.3) when $m=n=1$. We shall see that for this particular case the linearization of (4.1.3) is generically non-zero and nilpotent, so we can classify this problem using the singularity-theoretic framework of $\S 3$, designed to permit a non-trivial linearization.

\subsection{Reduction to a smaller domain}

We now investigate how the singularity theory for higher-dimensional problems (4.0.1), (4.0.2) reduces to an associated problem on a 1-dimensional domain. We assume without loss of generality that $1 \leqslant m \leqslant n$. We carry out the ideas for Case 1 of Theorem 4.2; the analogous result for Case 2 follows immediately by setting $b \equiv d \equiv 0$ in (4.1.2). We start by computing a set of generators for $\stackrel{\leftrightarrow}{\mathscr{E}} \mathbf{O}(2)^{N}\left(\operatorname{Fix} \mathbf{Z}_{2}^{N}\right)$.

\subsubsection{Generators of $\stackrel{\leftrightarrow}{\mathscr{E}} \mathbf{O}(2)^{N}\left(\right.$ Fix $\left.\mathbf{Z}_{2}^{N}\right)$}

As mentioned in $\$ 4.1$, computing generators for $\overrightarrow{\mathscr{E}}_{\mathbf{O}(2)^{N}}\left(\mathrm{Fix} \mathbf{Z}_{2}^{N}\right)$ by first computing generators for $\overrightarrow{\mathscr{E}}_{\mathbf{O}(2)^{N}}$ and then restricting those to Fix $\mathbf{Z}_{2}^{N}$ leads to very complicated calculations. The same kind of problem occurs for the module $\stackrel{\overleftrightarrow{\mathscr{E}}}{\mathbf{O}(2)^{N}}\left(\right.$ Fix $\left.\mathbf{Z}_{2}^{N}\right)$, and there we use a generating set for the invariants restricted to Fix $\mathbf{Z}_{2}^{N}$ to compute a set of generators for $\overleftrightarrow{\mathscr{E}}_{\mathbf{O}(2)^{N}}\left(\operatorname{Fix} \mathbf{Z}_{2}^{N}\right)$. Gomes and Stewart [14] use a simple calculation to establish the well-known fact that equivariants of torus actions can easily be derived from invariants. The essential point is that torus actions diagonalize over $\mathbb{C}$, so invariants are generated by monomials. We show that a similar approach leads, equally simply, to generators for the equivariant matrix germs. Explicitly, we have the following result, analogous to Theorem 4 of [14].

Theorem 4.3. Consider the action of $\mathbf{T}^{N}$ on $\mathbb{C}^{N}$ given by (4.0.3). With coordinates $\left(z_{1}, \bar{z}_{1}, \ldots, z_{2^{N-1}}, \bar{z}_{2^{N-1}}, \ldots, z_{2^{N}}, \bar{z}_{2^{N}}\right)$ on $\mathbb{C}^{N}$, the module $\overleftrightarrow{\mathscr{E}}_{\mathbf{T}^{N}}$ is 
generated over the ring of one-parameter $\mathbb{C}$-valued invariants by matrices $M=\left(M_{i j}\right)_{1 \leqslant i, j \leqslant 2^{N+1}}$ with monomial entries $M_{i j}$ satisfying the following conditions.

(A) Any generator $M=\left(M_{i j}\right)_{1 \leqslant i, j \leqslant 2^{N+1}}$ for $\stackrel{\overleftrightarrow{\mathscr{E}}}{\mathbf{T}^{N}}$ has two non-zero monomial entries, namely

$$
\begin{array}{ll}
M_{p q} \text { and } M_{p+1, q-1}=\overline{M_{p q}}, & \text { for } p \text { odd }, q \text { even }, \\
M_{p q} \text { and } M_{p+1, q+1}=\overline{M_{p q}}, & \text { for } p \text { odd }, q \text { odd } .
\end{array}
$$

(B) The conditions on the indices are:

$$
\begin{aligned}
i=j \Longrightarrow M_{i i} \in \mathscr{E}_{T^{N}}, & \begin{cases}\left(\frac{\bar{z}_{i+1}}{2} \frac{z_{j+1}}{2}\right)^{-1}\left(I_{\mu}^{\alpha} I_{\nu}^{\beta}\right) & \text { for } i \text { odd }, j \text { odd }, \\
\left(\frac{\bar{z}_{i+1}}{2} \frac{\bar{z}_{j}}{2}\right)^{-1}\left(I_{\mu}^{\alpha} I_{\nu}^{\beta}\right) & \text { for } i \text { odd }, j \text { even }, \\
\left(\frac{z_{i}}{2} \frac{z_{j+1}}{2}\right)^{-1}\left(I_{\mu}^{\alpha} I_{\nu}^{\beta}\right) & \text { for } i \text { even, } j \text { odd }, \\
\left(\frac{z_{i}}{2} \frac{\bar{z}_{j}}{2}\right)^{-1}\left(I_{\mu}^{\alpha} I_{\nu}^{\beta}\right) & \text { for } i \text { even, } j \text { even } .\end{cases}
\end{aligned}
$$

Here $I_{\mu}$ and $I_{\nu}$ are any two generators of $\mathscr{E}_{T^{N}}$ such that

$$
\begin{array}{ll}
\alpha=1, \quad \beta=0 & \text { if } m_{i} n_{j} \text { divides } I_{\mu}, \\
\alpha=2, \quad \beta=0 & \text { if } m_{i} n_{j} \text { divides } I_{\mu}^{2}, \\
\alpha=1, \quad \beta=1 & \text { if } m_{i} \text { divides } I_{\mu} \text { and } n_{j} \text { divides } I_{\nu},
\end{array}
$$

with $m_{i} n_{j}$ given by

$$
m_{i} n_{j}= \begin{cases}\left(\frac{\bar{z}_{i+1}}{2} \frac{z_{j+1}}{2}\right) & \text { for } i \text { odd }, j \text { odd } \\ \left(\frac{\bar{z}_{i+1}}{2} \frac{\bar{z}_{j}}{2}\right) & \text { for } i \text { odd }, j \text { even }, \\ \left(\frac{z_{i}}{2} \frac{z_{j+1}}{2}\right) & \text { for } i \text { even, } j \text { odd } \\ \left(\frac{z_{i}}{2} \frac{\bar{z}_{j}}{2}\right) & \text { for } i \text { even, } j \text { even } .\end{cases}
$$

Proof. The equalities (4.2.1) follow from the choice of coordinate system. All other conditions on the indices follow by direct computation. As an illustration we compute some of the $M_{i j}$ when $N=2$. We omit the parameter $\lambda$ to simplify the notation.

Any $\theta=\left(\theta_{1}, \theta_{2}\right) \in \mathbf{T}^{2}$ acts diagonally on $\mathbb{C}^{4}$ by (see (4.0.3))

$$
\begin{aligned}
\theta \cdot\left(z_{1}, z_{2}, z_{3}, z_{4}\right) \equiv & \left(\theta \cdot z_{1}, \theta \cdot z_{2}, \theta \cdot z_{3}, \theta \cdot z_{4}\right) \\
= & \left(\exp \left\{\left(\frac{k_{1}}{L_{1}} \theta_{1}+\frac{k_{2}}{L_{2}} \theta_{2}\right) i\right\} z_{1}, \exp \left\{\left(\frac{k_{1}}{L_{1}} \theta_{1}-\frac{k_{2}}{L_{2}} \theta_{2}\right) i\right\} z_{2},\right. \\
& \left.\quad \exp \left\{\left(\frac{l_{1}}{L_{1}} \theta_{1}+\frac{l_{2}}{L_{2}} \theta_{2}\right) i\right\} z_{3}, \exp \left\{\left(\frac{l_{1}}{L_{1}} \theta_{1}-\frac{l_{2}}{L_{2}} \theta_{2}\right) i\right\} z_{4}\right) .
\end{aligned}
$$


Consider $\quad z \in \mathbb{C}^{4}$ in coordinates $z=\left(z_{1}, \bar{z}_{1}, z_{2}, \bar{z}_{2}, z_{3}, \bar{z}_{3}, z_{4}, \bar{z}_{4}\right)$. A simple calculation implies that a general $\mathbb{C}$-valued monomial

$$
z_{1}^{\gamma_{1}} \bar{z}_{1}^{\delta_{1}} z_{2}^{\gamma_{2}} \bar{z}_{2}^{\delta_{2}} z_{3}^{\gamma_{3}} \bar{z}_{3}^{\delta_{3}} z_{4}^{\gamma_{4}} \bar{z}_{4}^{\delta_{4}}
$$

is $\mathbf{T}^{2}$-invariant if and only if

$$
\begin{aligned}
& k_{1}\left[\left(\gamma_{1}-\delta_{1}\right)+\left(\gamma_{2}-\delta_{2}\right)\right]+l_{1}\left[\left(\gamma_{3}-\delta_{3}\right)+\left(\gamma_{4}-\delta_{4}\right)\right]=0, \\
& k_{2}\left[\left(\gamma_{1}-\delta_{1}\right)-\left(\gamma_{2}-\delta_{2}\right)\right]+l_{2}\left[\left(\gamma_{3}-\delta_{3}\right)-\left(\gamma_{4}-\delta_{4}\right)\right]=0 .
\end{aligned}
$$

Recall that $M \in \overleftrightarrow{\mathscr{E}} \mathbf{T}^{2}$ if and only if $M(\theta z) \theta w=\theta M(z) w$ for all $z, w \in \mathbb{C}^{4}$ and all $\theta \in \mathbf{T}^{2}$. We now perform explicit computations of two typical cases, $M_{11}$ and $M_{14}$.

Consider the entry $M_{11}$ and assume it is non-zero. If we write

$$
M_{11}=z_{1}^{\alpha_{1}} \bar{z}_{1}^{\beta_{1}} z_{2}^{\alpha_{2}} \bar{z}_{2}^{\beta_{2}} z_{3}^{\alpha_{3}} \bar{z}_{3}^{\beta_{3}} z_{4}^{\alpha_{4}} \bar{z}_{4}^{\beta_{4}}
$$

then $M \in \overleftrightarrow{\mathscr{E}^{2}} \mathbf{T}^{2}$ if and only if $M_{11}(\theta z) \theta w_{1}=\theta M_{11}(z) w_{1}$; that is, if and only if

$$
\begin{aligned}
& k_{1}\left[\left(\alpha_{1}-\beta_{1}\right)+\left(\alpha_{2}-\beta_{2}\right)\right]+l_{1}\left[\left(\alpha_{3}-\beta_{3}\right)+\left(\alpha_{4}-\beta_{4}\right)\right]=0, \\
& k_{2}\left[\left(\alpha_{1}-\beta_{1}\right)-\left(\alpha_{2}-\beta_{2}\right)\right]+l_{2}\left[\left(\alpha_{3}-\beta_{3}\right)-\left(\alpha_{4}-\beta_{4}\right)\right]=0 .
\end{aligned}
$$

Comparing these equalities with (4.2.2) we find that $M_{11}$ is $\mathbf{T}^{2}$-invariant and (4.2.1) implies that $M_{22}=\overline{M_{11}}$.

Suppose that the entry $M_{14}$ is non-zero. Writing

$$
M_{14}=z_{1}^{\alpha_{1}} \bar{z}_{1}^{\beta_{1}} z_{2}^{\alpha_{2}} \bar{z}_{2}^{\beta_{2}} z_{3}^{\alpha_{3}} \bar{z}_{3}^{\beta_{3}} z_{4}^{\alpha_{4}} \bar{z}_{4}^{\beta_{4}}
$$

we have $M \in \overleftrightarrow{\mathscr{E}}_{\mathbf{T}^{2}}$ if and only if $M_{14}(\theta z) \theta \bar{w}_{2}=\theta M_{14}(z) \bar{w}_{2}$, that is, if and only if

$$
\begin{array}{r}
k_{1}\left[\left(\alpha_{1}-\beta_{1}\right)+\left(\alpha_{2}-\beta_{2}\right)\right]+l_{1}\left[\left(\alpha_{3}-\beta_{3}\right)+\left(\alpha_{4}-\beta_{4}\right)\right]-2 k_{1}=0, \\
k_{2}\left[\left(\alpha_{1}-\beta_{1}\right)-\left(\alpha_{2}-\beta_{2}\right)\right]+l_{2}\left[\left(\alpha_{3}-\beta_{3}\right)-\left(\alpha_{4}-\beta_{4}\right)\right]=0 .
\end{array}
$$

These conditions imply that $\bar{z}_{1} \bar{z}_{2} M_{14}$ is an invariant. From (4.2.1), we have $M_{23}=\overline{M_{14}}$.

By such calculations, we conclude that the following elements generate all the $\mathbf{T}^{2}$-invariant matrices:

$$
\begin{array}{rrrr}
M_{11}, & \bar{z}_{1} \bar{z}_{1} M_{12}, & \bar{z}_{1} z_{2} M_{13}, & \bar{z}_{1} \bar{z}_{2} M_{14}, \\
\bar{z}_{1} z_{3} M_{15}, & \bar{z}_{1} \bar{z}_{3} M_{16}, & \bar{z}_{1} z_{4} M_{17}, & \bar{z}_{1} \bar{z}_{4} M_{18}, \\
\bar{z}_{2} z_{1} M_{31}, & \bar{z}_{2} \bar{z}_{1} M_{32}, & M_{33}, & \bar{z}_{2} \bar{z}_{2} M_{34}, \\
\bar{z}_{2} z_{3} M_{35}, & \bar{z}_{2} \bar{z}_{3} M_{36}, & \bar{z}_{2} z_{4} M_{37}, & \bar{z}_{2} \bar{z}_{4} M_{38}, \\
\bar{z}_{3} z_{1} M_{51}, & \bar{z}_{3} \bar{z}_{1} M_{52}, & \bar{z}_{3} z_{2} M_{53}, & \bar{z}_{3} \bar{z}_{2} M_{54}, \\
M_{55}, & \bar{z}_{3} \bar{z}_{3} M_{56}, & \bar{z}_{3} z_{4} M_{57}, & \bar{z}_{3} \bar{z}_{4} M_{58}, \\
\bar{z}_{4} z_{1} M_{71}, & \bar{z}_{4} \bar{z}_{1} M_{72}, & \bar{z}_{4} z_{2} M_{73}, & \bar{z}_{4} \bar{z}_{2} M_{74}, \\
\bar{z}_{4} z_{3} M_{75}, & \bar{z}_{4} \bar{z}_{3} M_{76}, & M_{77}, & \bar{z}_{4} \bar{z}_{4} M_{78} .
\end{array}
$$

The other 32 remaining $M_{i j}$ are derived from (4.2.1). By inspection on all these elements we get the stated conditions on the indices. 


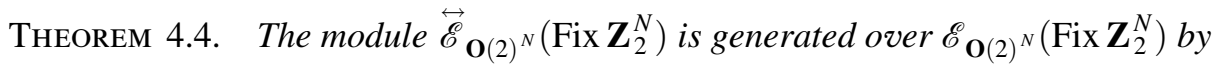

$$
\begin{aligned}
& \left\{1, x^{n-2} y^{m}\right\}\left(\begin{array}{ll}
I & 0 \\
0 & 0
\end{array}\right), \quad\left\{x y, x^{n-1} y^{m-1}\right\}\left(\begin{array}{ll}
0 & 0 \\
I & 0
\end{array}\right), \\
& \left\{x y, x^{n-1}, y^{m-1}\right\}\left(\begin{array}{ll}
0 & I \\
0 & 0
\end{array}\right), \quad\left\{1, x^{n} y^{m-2}\right\}\left(\begin{array}{ll}
0 & 0 \\
0 & I
\end{array}\right),
\end{aligned}
$$

where I represents the $2^{N-1} \times 2^{N-1}$ identity matrix.

If $1=m<n$ then all the generators are the same except the last, which becomes

$$
x^{n} y\left(\begin{array}{ll}
0 & 0 \\
0 & I
\end{array}\right) .
$$

Finally, if $m=n=1$ then the generators are

$$
\{1, x y\} \cdot\left\{\left(\begin{array}{ll}
I & 0 \\
0 & 0
\end{array}\right),\left(\begin{array}{ll}
0 & I \\
0 & 0
\end{array}\right),\left(\begin{array}{ll}
0 & 0 \\
I & 0
\end{array}\right),\left(\begin{array}{ll}
0 & 0 \\
0 & I
\end{array}\right)\right\} .
$$

Proof. This is a consequence of Theorems 4.2 and 4.3, as can be seen by observing that we can rewrite any non-diagonal matrix-germ generator defined on Fix $\mathbf{Z}_{2}^{N}$ as a diagonal matrix. Also, we do not need to symmetrize generators of $\overleftrightarrow{\mathscr{E}}_{\mathbf{T}^{N}}$ over the flips (4.0.4) of $\mathbf{Z}_{2}^{N}$ to get $\stackrel{\leftrightarrow}{\mathscr{E}} \mathbf{O}(2)^{N}\left(\right.$ Fix $\left.\mathbf{Z}_{2}^{N}\right)$, since $\mathbf{Z}_{2}^{N}$ acts trivially on Fix $\mathbf{Z}_{2}^{N}$.

\subsubsection{Singularity theory}

Let $g \in \overrightarrow{\mathscr{E}}_{\mathbf{O}(2)^{N}}\left(\operatorname{Fix} \mathbf{Z}_{2}^{N}\right)$, so that

$$
g(x, \ldots, x, y, \ldots, y, \lambda)=\left(\begin{array}{c}
a(u, v, \lambda) x+b(u, v, \lambda) x^{n-1} y^{m} \\
\vdots \\
a(u, v, \lambda) x+b(u, v, \lambda) x^{n-1} y^{m} \\
c(u, v, \lambda) y+d(u, v, \lambda) x^{n} y^{m-1} \\
\vdots \\
c(u, v, \lambda) y+d(u, v, \lambda) x^{n} y^{m-1}
\end{array}\right)
$$

where $u=x^{2}, v=y^{2}$.

The extended tangent space of $g$ is given by (see (3.2.8))

$$
T(g)=\mathscr{E}_{\mathbf{O}(2)^{N}}\left(\operatorname{Fix} \mathbf{Z}_{2}^{N}\right)\left\langle S_{j} g, 1 \leqslant j \leqslant 8,(d g) g_{i}, 1 \leqslant i \leqslant 4\right\rangle+\mathscr{E}_{\lambda} g_{\lambda} .
$$

The $S_{j}$ are the generators of $\stackrel{\mathscr{E}}{\mathbf{O}(2)^{N}}\left(\operatorname{Fix} \mathbf{Z}_{2}^{N}\right)$ given by Theorem 4.4 , and the $g_{i}$ are given by (4.1.1).

Here $(d g)$ is the $2^{N} \times 2^{N}$ matrix

$$
(d g)=\left(\begin{array}{ll}
\frac{\partial p}{\partial x} U & \frac{\partial p}{\partial y} U \\
\frac{\partial q}{\partial x} U & \frac{\partial q}{\partial y} U
\end{array}\right)
$$

where $U$ denotes the $2^{N-1} \times 2^{N-1}$ matrix with all entries equal to 1 , $p=a x+b x^{n-1} y^{m}$, and $q=c y+d x^{n} y^{m-1}$. 
Consider the isomorphism

$$
\begin{aligned}
\varphi: & \mathbb{R}^{2} \rightarrow \operatorname{Fix} \mathbf{Z}_{2}^{N}, \\
(x, y) & \mapsto(x, \ldots, x, y, \ldots, y),
\end{aligned}
$$

and associate to $g$ the corresponding term in $\overrightarrow{\mathscr{E}}_{\mathbf{O}(2)}\left(\operatorname{Fix} \mathbf{Z}_{2}\right)$ given by

$$
h(x, y, \lambda)=\left(\begin{array}{l}
a(u, v, \lambda) x+b(u, v, \lambda) x^{n-1} y^{m} \\
c(u, v, \lambda) y+d(u, v, \lambda) x^{n} y^{m-1}
\end{array}\right) .
$$

That is, $h=\left(\varphi^{-1}, I_{\lambda}\right) \circ g \circ\left(\varphi, I_{\lambda}\right)$. Hence $\overrightarrow{\mathscr{E}}_{\mathbf{O}(2)^{N}}\left(\right.$ Fix $\left.\mathbf{Z}_{2}^{N}\right)$ and $\overrightarrow{\mathscr{E}}_{\mathbf{O}(2)}\left(\right.$ Fix $\left.\mathbf{Z}_{2}\right)$ are isomorphic spaces. By the same argument, the extended tangent spaces of $g$ and $h$ are also isomorphic. Consequently, the codimension of a germ in 'pre-normal form' (by which we mean the germ on $\mathbb{R}^{2}$ defined by the two distinct entries in (4.1.2)) is independent of $N$.

The generators of $\stackrel{\leftrightarrow}{\mathscr{E}} \mathbf{O}(2)\left(\right.$ Fix $\left.\mathbf{Z}_{2}\right)$ found by Armbruster and Dangelmayr [2] are obtained by taking $I_{1 \times 1}=1 \in \mathbb{R}$ in the matrix germs that appear in Theorem 4.4, so $\overleftrightarrow{\mathscr{E}}_{\mathbf{O}(2)^{N}}\left(\right.$ Fix $\left.\mathbf{Z}_{2}^{N}\right)$ is isomorphic to $\overleftrightarrow{\mathscr{E}} \mathbf{O}(2)\left(\right.$ Fix $\left.\mathbf{Z}_{2}\right)$.

Finally, consider an equivalence relation on $\overleftrightarrow{\mathscr{E}}_{\mathbf{O}(2)^{N}}\left(\right.$ Fix $\left.\mathbf{Z}_{2}^{N}\right)$ as in Definition 3.2. A change of coordinates $\Psi$ on Fix $\mathbf{Z}_{2}^{N} \times \mathbb{R}$ can be taken as

$$
\Psi=\left(\varphi, I_{\lambda}\right) \circ \Phi \circ\left(\varphi^{-1}, I_{\lambda}\right),
$$

where $\Phi$ is a coordinate change on Fix $\mathbf{Z}_{2} \times \mathbb{R}$. Therefore, applying singularity theory to bifurcation problems defined on $\overrightarrow{\mathscr{E}}_{\mathbf{O}(2)^{N}}\left(\right.$ Fix $\left.\mathbf{Z}_{2}^{N}\right)$ reduces to the standard analysis of the 1-dimensional problem in $\overrightarrow{\mathscr{E}}_{\mathbf{O}(2)}\left(\operatorname{Fix} \mathbf{Z}_{2}\right)$ associated to it via the isomorphism (4.2.4).

\subsubsection{Alternative way to compute equivariant matrix germs}

Next we give a result on matrix germs in the equivariant context, which is useful for computing $\overleftrightarrow{\mathscr{E}}_{\Gamma}$, namely Proposition 4.5 below. We give an example to show how this result can be applied. The results we present here were motivated by initial attempts to obtain a corresponding result in the hidden symmetry context, particularly when trying to compute generators for $\stackrel{\leftrightarrow}{\mathscr{E}} \mathbf{O}(2)^{N}\left(\operatorname{Fix} \mathbf{Z}_{2}^{N}\right)$.

The classification of one-parameter $\Gamma$-equivariant germs defined on a vector space $V$, with $\Gamma$ a compact Lie group, involves matrix germs in the module

$$
\stackrel{\mathscr{E}}{\mathscr{E}}_{\Gamma}=\left\{S:(V \times \mathbb{R}, 0) \rightarrow \operatorname{Hom}(V, V): S(x, \lambda)=\gamma^{-1} S(\gamma x, \lambda) \gamma, \forall \gamma \in \Gamma, \forall x \in V\right\}
$$

If $x \in V$ then for $h \in \overrightarrow{\mathscr{E}}_{\Gamma}$ we compute $S(x, \lambda) h(\Phi(x, \lambda), \Lambda(\lambda))$, where $\Phi$ is a diffeomorphism germ in $\overrightarrow{\mathscr{E}}_{\Gamma}$ and $\Lambda$ is a diffeomorphism germ in $\mathscr{E}_{\lambda}$. Hence, we are interested in the product

$$
S(x, \lambda) g(x, \lambda)
$$

for $g(x, \lambda)=h(\Phi(x, \lambda), \Lambda(\lambda)) \in \overrightarrow{\mathscr{E}}_{\Gamma}$. It turns out that it is sometimes easier to compute generators for matrix germs in the larger module

$$
\mathscr{R}_{\Gamma}=\left\{R:(V \times \mathbb{R}, 0) \rightarrow \operatorname{Hom}(V, V): R g \in \overrightarrow{\mathscr{E}}_{\Gamma}, \forall g \in \overrightarrow{\mathscr{E}}_{\Gamma}\right\}
$$

as Example 4.7 below illustrates. In fact, the use of such matrix germs leads to 
the usual equivalence relation using $\overleftrightarrow{\mathscr{E}}_{\Gamma}$. To see why, introduce the space

$$
\mathscr{T}_{\Gamma}=\left\{T:(V \times \mathbb{R}, 0) \rightarrow \operatorname{Hom}(V, V): T(x, \lambda) g(x, \lambda)=0, \forall g \in \overrightarrow{\mathscr{E}}_{\Gamma}, \forall x \in V\right\} .
$$

We then have the following.

Proposition 4.5. Let $\Gamma$ be a compact Lie group acting linearly on $V$, and let $\mathscr{R}_{\Gamma}$ denote the module over $\mathscr{E}_{\Gamma}$ defined by (4.2.5). Then $\mathscr{R}_{\Gamma}$ can be decomposed as

$$
\mathscr{R}_{\Gamma}=\overleftrightarrow{\mathscr{E}}_{\Gamma}+\mathscr{T}_{\Gamma}
$$

Proof. To simplify the notation, omit $\lambda$.

It is easy to check that $\overleftrightarrow{\mathscr{E}}_{\Gamma}$ and $\mathscr{T}_{\Gamma}$ are subsets of $\mathscr{R}_{\Gamma}$.

Given $R \in \mathscr{R}_{\Gamma}$ and $x \in V$ we use the normalized Haar integral to define the averaging operator

$$
\widehat{R}(x)=\int_{\Gamma} \gamma^{-1} R(\gamma x) \gamma d \gamma
$$

It is straightforward to show that $R \mapsto \widehat{R}$ is a projection onto $\overleftrightarrow{\mathscr{E}}_{\Gamma}$. Hence

$$
\mathscr{R}_{\Gamma}=\overleftrightarrow{\mathscr{E}}_{\Gamma} \oplus \operatorname{ker}(R \mapsto \widehat{R})
$$

Finally, elements in the kernel define matrix germs which annihilate equivariant vector fields. In fact, let $K \in \operatorname{ker}(R \mapsto \widehat{R})$, that is, $\widehat{K} \equiv 0$. For any $g \in \overrightarrow{\mathscr{E}}_{\Gamma}$,

$$
\int_{\Gamma} \gamma^{-1} \gamma K(x) g(x) d \gamma=\int_{\Gamma} \gamma^{-1} K(\gamma x) \gamma g(x) d \gamma=\left(\int_{\Gamma} \gamma^{-1} K(\gamma x) \gamma d \gamma\right) g(x)=0,
$$

and since $\int_{\Gamma} d \gamma=1$,

$$
K(x) g(x)=0 .
$$

Hence, $K \in \mathscr{T}_{\Gamma}$. Therefore, $\operatorname{ker}(R \mapsto \widehat{R}) \subseteq \mathscr{T}_{\Gamma}$.

REMARK 4.6. If $\left\{g_{1}, \ldots, g_{s}\right\}$ is a set of generators of $\overrightarrow{\mathscr{E}}_{\Gamma}$, then for all $R \in \mathscr{R}_{\Gamma}$,

$$
R g_{i}=\sum_{j=1}^{s} f_{i j} g_{j}, \quad \text { for all } i=1, \ldots, s, \text { with } f_{i j} \in \mathscr{E}_{\Gamma} .
$$

By Proposition 4.5, we can decompose $R=S_{R}+T_{R}$, where $S_{R} \in \overleftrightarrow{\mathscr{E}}_{\Gamma}$ and $T_{R} \in \mathscr{T}_{\Gamma}$. So

$$
R g_{i}=S_{R} g_{i}+T_{R} g_{i}=S_{R} g_{i}, \quad \text { for all } i=1, \ldots, s .
$$

Therefore, for all $x \in V$,

$$
R(x) g_{i}(x)=S_{R}(x) g_{i}(x) T_{R}(x) g_{i}(x)=S_{R}(x) g_{i}(x),
$$

since $T_{R}(x) g_{i}(x)=0$, by (4.2.6). In other words, germs that are equivalent using matrices in $\mathscr{R}_{\Gamma}$ are actually equivalent using matrices in $\overleftrightarrow{\mathscr{E}}_{\Gamma}$. The converse is also true, since $\overleftrightarrow{\mathscr{E}}_{\Gamma} \subseteq \mathscr{R}_{\Gamma}$. Hence, the set of generators of $\mathscr{R}_{\Gamma}$ satisfying (4.2.9) that are in the module $\overleftrightarrow{\mathscr{E}}_{\Gamma}$ are all the elements in $\overleftrightarrow{\mathscr{E}}_{\Gamma}$ needed to compute normal forms in the classification problem. Note, however, that we are not claiming that $\mathscr{R}_{\Gamma}=\overleftrightarrow{\mathscr{E}}_{\Gamma}$. This would be the case if

$$
R(x) g_{i}(y)=S_{R}(x) g_{i}(y), \quad \text { for all } x, y \in V,
$$

but this does not follow from (4.2.7). 
Example 4.7. Consider the standard action of $\mathbf{O}(2)$ on $\mathbb{C}$ given by

$$
\theta \cdot z=e^{i \theta} z \text { for } 0 \leqslant \theta<2 \pi \text { and } \kappa \cdot z=\bar{z} \text {. }
$$

By [13] there is one equivariant generator $g_{1}(z, \lambda)=z$ and one invariant generator $u=z \bar{z}$. With coordinates $(z, \bar{z})$ on $\mathbb{C}$, any $R \in \mathscr{R}_{\mathbf{O}(2)}$ can be expressed as

$$
R(z, \lambda) w=a(z, \lambda) w+b(z, \lambda) \bar{w}
$$

where

$$
a(z, \lambda)=\sum_{i \geqslant 0} \alpha_{i} z^{i}+\sum_{j \geqslant 1} \beta_{j} \bar{z}^{j}, \quad b(z, \lambda)=\sum_{\kappa \geqslant 0} \gamma_{\kappa} z^{\kappa}+\sum_{\ell \geqslant 1} \delta_{\ell} \bar{z}^{\ell}
$$

with $\alpha_{i}, \beta_{j}, \gamma_{\kappa}, \delta_{\ell} \in \mathscr{E}_{\mathbf{O}(2)}$. From (4.2.9) we have $R(z, \lambda) z=f_{11}(u, \lambda) z$, where $f_{11} \in \mathscr{E} \mathbf{O}(2)$, that is, $R(z, \lambda) z=A_{0} z+A_{1} u z+A_{2} u^{2} z+\ldots$ where $A_{j} \in \mathbb{R}$ for all $j$. Uniqueness of Taylor expansion implies that

$$
R(z, \lambda) z=\left(\alpha_{0}+\gamma_{2} u\right) z
$$

Write (4.2.10) with these coefficients:

$$
w \mapsto \alpha_{0} w+\gamma_{2} z^{2} \bar{w}
$$

It is now straightforward that $w \mapsto w$ and $w \mapsto z^{2} \bar{w}$ are both in $\overleftrightarrow{\mathscr{E}}_{\mathbf{O}(2)}$. Therefore, these are the generators of $\stackrel{\mathscr{E}}{\mathbf{O}(2)}$ that appear in the classification of $\mathbf{O}(2)$ equivariant bifurcation problems. Notice that $w \mapsto u w-z^{2} \bar{w} \in \overleftrightarrow{\mathscr{E}}_{\mathbf{O}(2)} \cap \mathscr{T}_{\mathbf{O}(2)}$, so this example shows that (4.2.7) may not be a direct sum.

\subsection{Two-mode interactions when $m=n=3$}

As mentioned earlier, the bifurcation analysis of (4.1.3) can be found in [2] for the cases when $m$ and $n$ are coprime. However, $m$ and $n$ can no longer be assumed coprime when $N>1$. In particular, the case $m=n=3$ requires further analysis, since the geometry of the associated bifurcation diagrams has interesting features. We consider this case here, studying the least degenerate bifurcation behaviour of zeros of (4.1.3). As proved in $\$ 4.2$, up to isomorphism the singularity theory that we are considering applied to bifurcation problems on $\mathrm{N}$ dimensional domains does not depend on $N$, so we can use the singularity theory of $\S 3$ and restrict the analysis for $N=1$. We therefore consider a germ $g \in \overrightarrow{\mathscr{E}}_{\mathbf{O}(2)}\left(\right.$ Fix $\left.\mathbf{Z}_{2}\right)$ which has the form

$$
g(x, y, \lambda)=\left(a(u, v, \lambda) x+b(u, v, \lambda) x^{2} y^{3}, c(u, v, \lambda) y+d(u, v, \lambda) x^{3} y^{2}\right)
$$

with $u=x^{2}, v=y^{2}$. Simplify notation by writing $g=[a, b, c, d]$. In contrast to the bifurcation analysis, the condition $\operatorname{gcd}(m, n) \neq 1$ does not affect the singularity theory, so the least degenerate normal form and its unfolding can be obtained from [2]. For completeness we derive it here, together with its unfolding. All computations were performed with a Maple program.

Assume that $g$ in (4.3.1) satisfies the following non-degeneracy conditions at $(x, y, \lambda)=(0,0,0)$ :

$$
\begin{gathered}
a_{u} \neq 0, \quad a_{\lambda} \neq 0, \quad c_{v} \neq 0, \quad c_{\lambda} \neq 0, \quad d \neq 0, \\
a_{u} c_{v}-c_{v} a_{u} \neq 0, \quad a_{u} c_{\lambda}-a_{\lambda} c_{u} \neq 0, \quad a_{v} c_{\lambda}-a_{\lambda} c_{v} \neq 0 .
\end{gathered}
$$

The inequalities above imply that the submodule $\mathscr{S}=\left[\mathscr{M}^{2}, \mathscr{M}, \mathscr{M}^{2}, \mathscr{M}\right]$ is 
contained in the unipotent tangent space $\mathrm{T}(g, \mathscr{U})$. It is easy to check that $\mathscr{S}$ is intrinsic, so in fact $\mathscr{S} \subseteq \operatorname{ItrT}(g, \mathscr{U})$. Therefore all germs in $\mathscr{S}$ are higher-order terms for $g$ (see (3.3.3)).

By performing changes of coordinates modulo $\mathscr{S}$ we find the normal form

$$
h(u, v, \lambda)=\left(\left(\varepsilon_{1} u+\rho_{1} v+\varepsilon_{2} \lambda\right) x+\mu_{1} x^{2} y^{3},\left(\kappa_{1} u+\varepsilon_{3} v+\varepsilon_{4} \lambda\right) y+\varepsilon_{5} x^{3} y^{2}\right)
$$

where

$$
\varepsilon_{1}=\frac{a_{u}}{\left|a_{u}\right|}, \quad \varepsilon_{2}=\frac{a_{\lambda}}{\left|a_{\lambda}\right|}, \quad \varepsilon_{3}=\frac{c_{v}}{\left|c_{v}\right|}, \quad \varepsilon_{4}=\frac{c_{\lambda}}{\left|c_{\lambda}\right|}, \quad \varepsilon_{5}=\frac{d}{|d|}
$$

and $\rho_{1}, \kappa_{1}$ and $\mu_{1}$ are the modal parameters

$$
\rho_{1}=\frac{\left|c_{\lambda}\right| a_{v}}{\left|a_{\lambda}\right|\left|c_{v}\right|}, \quad \kappa_{1}=\frac{\left|a_{\lambda}\right| c_{u}}{\left|a_{u}\right|\left|c_{\lambda}\right|}, \quad \mu_{1}=\frac{\left|a_{u}\right|\left|c_{\lambda}\right|^{2} b}{\left|c_{v}\right||d|\left|a_{\lambda}\right|^{2}} .
$$

The extended tangent space $\mathrm{T}(h)$ is generated modulo $\mathscr{S}$ by

$$
\begin{gathered}
{\left[\varepsilon_{1} u+\rho_{1} v+\varepsilon_{2} \lambda, \mu_{1}, 0,0\right], \quad\left[0,0, \kappa_{1} u+\varepsilon_{3} v+\varepsilon_{4} \lambda, \varepsilon_{5}\right],} \\
{\left[3 \varepsilon_{1} u+\rho_{1} v+\varepsilon_{2} \lambda, 2 \mu_{1}, 2 \kappa_{1} u, 3 \varepsilon_{5}\right],\left[2 \rho_{1} v, 3 \mu_{1}, \kappa_{1} u+3 \varepsilon_{3} v+\varepsilon_{4} \lambda, 2 \varepsilon_{5}\right],} \\
{\left[\varepsilon_{2}, 0, \varepsilon_{4}, 0\right], \quad\left[\varepsilon_{2} \lambda, 0, \varepsilon_{4} \lambda, 0\right] .}
\end{gathered}
$$

If we write these elements as linear combinations of $[1,0,0,0],[u, 0,0,0]$, $[v, 0,0,0],[\lambda, 0,0,0],[0,1,0,0],[0,0,1,0],[0,0, u, 0],[0,0, v, 0],[0,0, \lambda, 0]$, $[0,0,0,1]$, we find a $6 \times 10$ matrix with rank 6 . Adding the rows $[1,0,0,0]$, $[v, 0,0,0],[0,1,0,0],[0,0, u, 0]$, we find a $10 \times 10$ invertible matrix. Hence these four germs form a basis for the complement to $\mathrm{T}(h)$ in $\overrightarrow{\mathscr{E}}_{\mathbf{O}(2)}\left(\mathrm{Fix} \mathbf{Z}_{2}\right)$. Thus the codimension of $h$ is 4 and its unfolding is

$$
H=\left(\left(\varepsilon_{1} u+\rho v+\varepsilon_{2} \lambda+\alpha\right) x+\mu x^{2} y^{3},\left(\kappa u+\varepsilon_{3} v+\varepsilon_{4} \lambda\right) y+\varepsilon_{5} x^{3} y^{2}\right)
$$

where $\rho \sim \rho_{1}, \kappa \sim \kappa_{1}, \mu \sim \mu_{1}, \alpha \sim 0$.

We now present the bifurcation analysis of zeros of the unfolding $H$. The solution branches for

$$
\left\{\begin{array}{l}
\left(\varepsilon_{1} u+\rho v+\varepsilon_{2} \lambda+\alpha\right) x+\mu x^{2} y^{3}=0 \\
\left(\kappa u+\varepsilon_{3} v+\varepsilon_{4} \lambda\right) y+\varepsilon_{5} x^{3} y^{2}=0
\end{array}\right.
$$

are

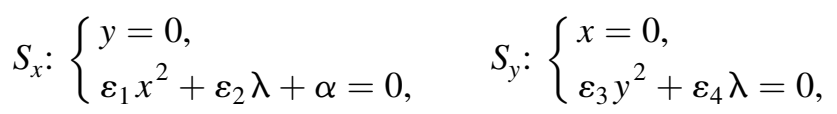

which we call pure-mode branches, and

$$
S_{m}:\left\{\begin{array}{l}
\varepsilon_{1} x^{2}+\rho y^{2}+\varepsilon_{2} \lambda+\mu x y^{3}+\alpha=0, \\
\kappa x^{2}+\varepsilon_{3} y^{2}+\varepsilon_{4} \lambda+\varepsilon_{5} x^{3} y=0
\end{array}\right.
$$

which we call a mixed-mode branch.

We now analyse the nature of secondary bifurcations for (4.3.2), that is, points different from the origin where the mixed-mode branch intersects a pure-mode branch. To do so, recall that a solution branch is transcritical at $\lambda=\lambda_{0}$ if it can be locally parametrized by $\lambda=\lambda(t)$, for $t$ near zero, with $\lambda_{0}=\lambda(0)$ and 
$\lambda^{\prime}(0) \neq 0$. The branch is subcritical if $t \lambda^{\prime}(t)<0$ for all non-zero $t$ near 0 , and supercritical if $t \lambda^{\prime}(t)>0$ for all non-zero $t$ near 0 .

Choose $\varepsilon_{1}=\varepsilon_{3}=\varepsilon_{5}=1, \quad \varepsilon_{2}=\varepsilon_{4}=-1, \kappa<1, \rho>1, \quad \alpha<0$ so that both $S_{x} \cap S_{m}$ and $S_{y} \cap S_{m}$ are non-empty and given by

$$
\begin{aligned}
& S_{x} \cap S_{m}=\left\{\left(x_{1}= \pm \sqrt{\frac{-\alpha}{1-\kappa}}, y_{1}=0, \lambda_{1}=\frac{-\kappa \alpha}{1-\kappa}\right)\right\}, \\
& S_{y} \cap S_{m}=\left\{\left(x_{2}=0, y_{2}= \pm \sqrt{\frac{\alpha}{1-\rho}}, \lambda_{2}=\frac{\alpha}{1-\rho}\right)\right\} .
\end{aligned}
$$

Project the zeros of (4.3.2) onto the $x, y$-plane (by eliminating $\lambda$ from the equations) to get

$$
F(x, y)=(1-\kappa) x^{2}+(\rho-1) y^{2}+\mu x y^{3}-x^{3} y+\alpha=0 .
$$

Considering $\lambda$ in a neighbourhood of $\lambda_{1}$ (see (4.3.3)), we have $F_{y}\left(x_{1}, y_{1}\right)=-x_{1}^{3} \neq 0$. Hence $\lambda$ can be locally parametrized by $x$, and

$$
\frac{d \lambda}{d x}\left(x_{1}\right)=2 x_{1} \kappa \neq 0
$$

Repeat for $\lambda$ in a neighbourhood of $\lambda_{2}$ (see (4.3.4)), $F_{x}\left(x_{2}, y_{2}\right)=\mu y_{2}^{3} \neq 0$. Then we see that $\lambda$ can be locally parametrized by $y$, and

$$
\frac{d \lambda}{d y}\left(y_{2}\right)=2 y_{2} \neq 0 \text {. }
$$

Now (4.3.5) and (4.3.6) imply that $S_{m}$ is transcritical at all points of secondary bifurcation. This is the fact that we want to emphasize: as pointed out by Armbruster and Dangelmayr [2], when $\operatorname{gcd}(m, n)=1$, not all secondary bifurcations are transcritical, and $S_{m}$-branches always develop subcritical or supercritical bifurcations. Now, points where $S_{m}$ is subcritical or supercritical are of pitchfork type for (4.3.2), as are all secondary bifurcations for $\mathbf{Z}_{2} \oplus \mathbf{Z}_{2}$-symmetric problems. Since (4.3.1) represents a $\mathbf{Z}_{2} \oplus \mathbf{Z}_{2}$-symmetric problem for $b \equiv d \equiv 0$, we can say that the fifth-order terms in (4.3.2) break all the $\mathbf{Z}_{2} \oplus \mathbf{Z}_{2}$-symmetry, since they are the terms in the equations that make $S_{m}$ transcritical at secondary bifurcations.

\subsection{Germs with a non-trivial linearization}

We now illustrate the singularity-theoretic techniques developed in this paper by considering the above types of mode interaction when the linearization is non-trivial.

We start by analysing the possible $\mathbf{O}(2)^{N}$-equivariant vector fields that may appear for different values of the mode vectors $\mathbf{k}$ and $\boldsymbol{\ell}$. The action of $\mathbf{O}(2)^{N}$ on $\mathbb{C}^{2^{N-1}}$ defined in (4.0.16) and (4.0.17) is absolutely irreducible, that is, the only $2^{N-1} \times 2^{N-1}$ matrices commuting with this action are multiples of the identity. Absolute irreducibility implies irreducibility, but the converse is false; see [13]. Let $A$ be a matrix of order $2^{N-1}$ commuting with $\mathbf{O}(2)^{N}$. The $\varepsilon_{j}$ in (4.0.16) satisfy $\varepsilon_{i} \neq \varepsilon_{j}$ if $i \neq j$, since the $a_{j}$ are all non-zero. Therefore, $A$ is of the form

$$
A=\left(\begin{array}{cccc}
A_{1} & & & \\
& A_{2} & & \\
& & \ddots & \\
0 & & A_{2^{N-2}}
\end{array}\right)
$$


where the $A_{j}$ are $2 \times 2$ matrices. Commutativity of $A$ with $\mathbf{Z}_{2}^{N}$ implies that all $A_{j}$ are multiples of the identity, and equal. Therefore the action of $\mathbf{O}(2)^{N}$ on $\mathbb{C}^{2^{N-1}}$ is in fact absolutely irreducible.

For the two-mode interaction problem $\widetilde{g}:\left(\mathbb{C}^{2^{N}} \times \mathbb{R}, 0\right) \rightarrow \mathbb{C}^{2^{N}}$, with $\widetilde{g}$ an $\mathbf{O}(2)^{N}$-equivariant germ, let $M$ be its linearization at the origin, that is, $(d \widetilde{g})_{0,0}=M$. Then $M$ is of order $2^{N}$ and commutes with $\mathbf{O}(2)^{N}$. If $k_{j} \neq \ell_{j}$ for some $j$, then the actions of $\theta$ on each direction are all different, and are like $2^{N}$ distinct plane rotations. So $M$ is given by two distinct copies $A^{1}$ and $A^{2}$ of the form (4.4.1). Now $M$ commutes with $\mathbf{Z}_{2}^{N}$, so $A^{1}$ and $A^{2}$ are both multiples of the identity. Therefore,

$$
M=\left(\begin{array}{cc}
c_{1} I & 0 \\
0 & c_{2} I
\end{array}\right)
$$

for some $c_{1}, c_{2} \in \mathbb{R}$. But $M$ has only zero eigenvalues, so $M \equiv 0$.

On the other hand, if $k_{j}=\ell_{j}(=1)$ for all $j=1, \ldots, N$, then $M$ decomposes into four copies of the form (4.4.1), and commutativity with $\mathbf{Z}_{2}^{N}$ implies that $M$ has the form

$$
M=\left(\begin{array}{ll}
c_{1} I & c_{2} I \\
c_{3} I & c_{4} I
\end{array}\right)
$$

for some $c_{1}, \ldots, c_{4} \in \mathbb{R}$. The zero-eigenvalue condition implies that $M$ is generically non-zero and nilpotent, with Jordan canonical form

$$
\left(\begin{array}{ll}
0 & I \\
0 & 0
\end{array}\right) \text {. }
$$

In the next subsection we apply the results of $\S 3$ to study the bifurcation of zeros of germs $\widetilde{g}$ with linearization (4.4.2) when restricted to the subspace Fix $\mathbf{Z}_{2}^{N}$. As proved in $\S 4.2$, we can study this case by assuming $N=1$.

\subsubsection{Singularity theory}

We now concentrate on the case $N=1$ to study the particular case $m=n=1$. The problem is to study the zeros of one-parameter bifurcation problems (4.1.2) given by

$$
g(x, y, \lambda)=(a x+b y, c y+d x)
$$

where $a, b, c$ and $d$ are germs of functions of $u=x^{2}, v=y^{2}$ and $\lambda$. The module of germs of the form (4.4.3) is $\overrightarrow{\mathscr{E}}_{\mathbf{O}(2)}\left(\right.$ Fix $\left.\mathbf{Z}_{2}\right)$, with generators

$$
e_{1}=\left(\begin{array}{l}
x \\
0
\end{array}\right), \quad e_{2}=\left(\begin{array}{l}
y \\
0
\end{array}\right), \quad e_{3}=\left(\begin{array}{l}
0 \\
y
\end{array}\right), \quad e_{4}=\left(\begin{array}{l}
0 \\
x
\end{array}\right) .
$$

We are interested in the bifurcation analysis of zeros of (4.4.3) when $(d g)_{0,0}$ is equal to

$$
L=\left(\begin{array}{ll}
0 & 1 \\
0 & 0
\end{array}\right)
$$

REMARK 4.8. From the usual point of view of steady-state bifurcation, since (4.4.5) is non-zero, we could perform a Liapunov-Schmidt reduction onto $\operatorname{ker} L$ and describe such bifurcations using a single variable. This already suggests a simple analysis concerning bifurcation of steady states; for example, secondary 
bifurcations are not to be expected, as we verify in $\$ 4.4 .2$. However, for theoretical purposes we choose this example to demonstrate a singularity-theoretic framework to study a 2-dimensional problem with a non-trivial linearization.

We now formulate $L$-contact equivalence in $\overrightarrow{\mathscr{E}}_{\mathbf{O}(2)}\left(\right.$ Fix $\left.\mathbf{Z}_{2}\right)$. Expression (4.2.3) gives generators for $\overleftrightarrow{\mathscr{E}}_{\mathbf{O}(2)}\left(\mathrm{Fix} \mathbf{Z}_{2}\right)$ :

$$
\begin{aligned}
& T_{1}=\left(\begin{array}{ll}
1 & 0 \\
0 & 0
\end{array}\right), \quad T_{2}=\left(\begin{array}{ll}
0 & 0 \\
0 & 1
\end{array}\right), \quad T_{3}=\left(\begin{array}{cc}
0 & x y \\
0 & 0
\end{array}\right), \quad T_{4}=\left(\begin{array}{cc}
0 & 0 \\
x y & 0
\end{array}\right), \\
& T_{5}=\left(\begin{array}{ll}
x y & 0 \\
0 & 0
\end{array}\right), \quad T_{6}=\left(\begin{array}{ll}
0 & 1 \\
0 & 0
\end{array}\right), \quad T_{7}=\left(\begin{array}{ll}
0 & 0 \\
1 & 0
\end{array}\right), \quad T_{8}=\left(\begin{array}{cc}
0 & 0 \\
0 & x y
\end{array}\right) .
\end{aligned}
$$

As in Example $3.4, h$ is $L$-contact equivalent to $g$ if there exist a matrixvalued germ $S \in \overleftrightarrow{\mathscr{E}}_{\mathbf{O}(2)}\left(\right.$ Fix $\left.\mathbf{Z}_{2}\right)$, a germ $\Phi \in \overrightarrow{\mathscr{E}}_{\mathbf{O}(2)}\left(\right.$ Fix $\left.\mathbf{Z}_{2}\right)$ and $\Lambda \in \mathscr{E}_{\lambda}$ satisfying (3.1.9)-(3.1.12) such that

$$
h(x, y, \lambda)=S(x, y, \lambda) g(\Phi(x, y, \lambda), \Lambda(\lambda)) .
$$

The group $\mathscr{K}_{L}$ of $L$-contact equivalences is

$$
\begin{array}{r}
\mathscr{K}_{L}=\left\{(S, \Phi, \Lambda) \in \overleftrightarrow{\mathscr{E}}_{\mathbf{O}(2)}\left(\operatorname{Fix} \mathbf{Z}_{2}\right) \times \overrightarrow{\mathscr{E}}_{\mathbf{O}(2)}\left(\operatorname{Fix} \mathbf{Z}_{2}\right) \times \mathscr{E}_{\lambda}:\right. \\
S, \Phi, \Lambda \text { satisfy }(3.1 .9)-(3.1 .12)\} .
\end{array}
$$

By (3.2.13) the restricted tangent space of $g$ is

$$
\begin{aligned}
\operatorname{RT}(g)=\{S g+(d g) \Phi: S \in \overleftrightarrow{\mathscr{E}} \mathbf{O}(2) & \left(\operatorname{Fix} \mathbf{Z}_{2}\right), \Phi \in \overrightarrow{\mathscr{E}}_{\mathbf{O}(2)}\left(\operatorname{Fix} \mathbf{Z}_{2}\right) \\
S(0) & \left.=\left(\begin{array}{cc}
\alpha & \beta \\
0 & \delta
\end{array}\right),(d \Phi)_{0}=\left(\begin{array}{cc}
A_{0} & B_{0} \\
0 & C_{0}
\end{array}\right), \alpha+C_{0}=0\right\} .
\end{aligned}
$$

We now give generators for $\mathrm{RT}(g)$ and for $\mathrm{T}(g)$. From (4.4.7), $\mathrm{RT}(g)$ is the module over $\mathscr{E}_{\mathbf{O}(2)}\left(\right.$ Fix $\left.\mathbf{Z}_{2}\right)$ generated by $T_{i} g, i=1, \ldots, 8, i \neq 1,7, \mathscr{M} T_{1} g, \mathscr{M} T_{7} g$, $(d g) e_{i}, i=1,2, \mathscr{M}(d g) e_{j}, j=3,4$, and $T_{1} g-(d g) e_{3}$, with $e_{i}$ given by (4.4.4) and $T_{j}$ given by (4.4.6). Now we denote $g(x, y, \lambda)=(a x+b y, c y+d x)$ by $[a, b, c, d]$ to list the generators of $\operatorname{RT}(g)$ :

$$
\begin{gathered}
\mathscr{M}[a, b, 0,0],[0,0, c, d], \quad[c v, d u, 0,0], \quad[0,0, a u, b v], \\
{[b v, a u, 0,0], \quad[d, c, 0,0], \mathscr{M}[0,0, b, a],[0,0, d u, c v],} \\
{\left[a+2 a_{u} u, 2 b_{u} u, 2 c_{u} u, d+2 d_{u} u\right], \quad \mathscr{M}\left[2 a_{v} v, b+2 b_{v} v, c+2 c_{v} v, 2 d_{v} v\right],} \\
{\left[2 b_{u} v, a+2 a_{u} u, d+2 d_{u} u, 2 c_{u} v\right], \quad \mathscr{M}\left[b+2 b_{v} v, 2 a_{v} u, 2 d_{v} u, c+2 c_{v} v\right],} \\
{\left[2 a_{v} v-a, 2 b_{v} v, c+2 c_{v} v, 2 d_{v} v\right] .}
\end{gathered}
$$

Expressions (3.2.8) and (4.4.8) imply that

$$
\begin{aligned}
\mathrm{T}(g)= & \mathrm{RT}(g)+\mathscr{E}_{\lambda} g_{\lambda} \\
& +\mathbb{R}\left\{[a, b, 0,0],[0,0, b, a],\left[b+2 b_{v} v, 2 a_{v} u, 2 d_{v} u, c+2 c_{v} v\right]\right\} .
\end{aligned}
$$




\subsubsection{Normal forms}

The results of the previous subsection lead to normal forms for bifurcation problems $g \in \overrightarrow{\mathscr{E}}_{\mathbf{O}(2)}\left(\right.$ Fix $\left.\mathbf{Z}_{2}\right)$ with nilpotent linearization (4.4.5). As we see below, the normal forms as well as their unfoldings can all be expressed in the form

$$
h(x, y, \lambda)=(y, f(u, 0, \lambda) x) .
$$

So $\operatorname{tr}(d h) \equiv 0$ on solutions, and the eigenvalues $\xi$ are the solutions of $\xi^{2}+\operatorname{det}(d h)=0$. Therefore, eigenvalues always occur in pairs $\pm \mu$, and there are values of $\lambda$ for which they are either real with opposite sign or purely imaginary. If the trivial solution is unstable for $\lambda<0$, then at a bifurcation point a change in the eigenvalues is generically as in Figure 1. In this case, solutions bifurcating supercritically are unstable, and those bifurcating subcritically have a pair of purely imaginary eigenvalues (non-hyperbolic equilibria). Notice that this situation is preserved under equivalence.

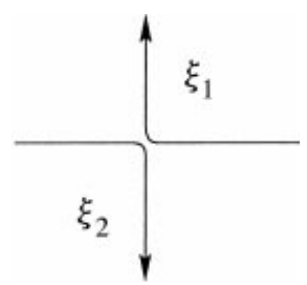

FIGURE 1. Generic change in the eigenvalues for the trivial solution when $\lambda$ crosses zero.

In the next proposition we present the generic normal form, two normal forms of codimension 1 and another more degenerate normal form with codimension 3 and modality 1 .

Proposition 4.9. Let $g(x, y, \lambda)=(p(u, v, \lambda) x+q(u, v, \lambda) y, \quad r(u, v, \lambda) y+$ $s(u, v, \lambda) x)$ be a bifurcation problem in $\overrightarrow{\mathscr{E}}_{\mathbf{O}(2)}\left(\right.$ Fix $\left.\mathbf{Z}_{2}\right)$ where $(d g)_{0,0}$ is the nilpotent linearization

$$
L=\left(\begin{array}{ll}
0 & 1 \\
0 & 0
\end{array}\right) .
$$

If $g$ satisfies the recognition conditions in Table 1 for the normal form $n_{j}$ for some $j=1, \ldots, 4$, then $g$ is equivalent to $n_{j}$.

Proof. The computations for each case are extensive and similar, so we describe them in detail for $n_{4}$ only, which involves the most extensive computations. The procedure basically consists of three steps. First find (some of) the higher-order terms. Then perform coordinate changes to remove further terms of high order and to normalise others. At this stage we seek a simple expression as normal form. Finally, compute the codimension and unfold this normal form. All the computations described below were performed using Maple.

Step 1. Write down a set of generators for the restricted tangent space $\operatorname{RT}\left(n_{4}\right)$ :

$$
\begin{aligned}
\operatorname{RT}\left(n_{4}\right)= & \left\langle[0, u, 0,0],[0, v, 0,0],[0, \lambda, 0,0],\left[0,0,0, \varepsilon_{1} u^{2}-\varepsilon_{2} \lambda^{2}+\mu u \lambda\right],[0,0,0, v],\right. \\
& {[v, 0,0,0],\left[\varepsilon_{1} u^{2}-\varepsilon_{2} \lambda^{2}+\mu u \lambda, 0,0,0\right],[0,0, u, 0],[0,0, v, 0],[0,0, \lambda, 0], } \\
& {\left.\left[0,0,0,5 \varepsilon_{1} u^{2}-\varepsilon_{2} \lambda^{2}+3 u \lambda\right],[u, 0,0,0],[\lambda, 0,0,0]\right\rangle . }
\end{aligned}
$$


TABLE 1. Normal forms in $\overrightarrow{\mathscr{E}}_{\mathbf{O}(2)}\left(\mathrm{Fix} \mathbf{Z}_{2}\right)$ and their classification. All derivatives are calculated at the origin, and $\varepsilon_{1}, \varepsilon_{2}= \pm 1$ with signs given by the corresponding derivatives.

\begin{tabular}{|c|c|c|c|c|}
\hline \multicolumn{5}{|c|}{$\begin{array}{l}\quad \text { Normal forms } \\
n_{1}=\left(y,\left(\varepsilon_{1} u-\varepsilon_{2} \lambda\right) x\right) \\
n_{2}=\left(y,\left(\varepsilon_{1} u-\varepsilon_{2} \lambda^{2}\right) x\right) \\
n_{3}=\left(y,\left(\varepsilon_{1} u^{2}-\varepsilon_{2} \lambda\right) x\right) \\
n_{4}=\left(y,\left(\varepsilon_{1} u^{2}-\varepsilon_{2} \lambda^{2}+\mu u \lambda\right) x\right)\end{array}$} \\
\hline \multicolumn{5}{|c|}{ Data } \\
\hline form & Recognition & $\varepsilon_{1}$ & $\varepsilon_{2}$ & terms \\
\hline$n_{1}$ & $s_{u}, s_{\lambda} \neq 0$ & $s_{u}$ & $-s_{\lambda}$ & \\
\hline$n_{2}$ & $\begin{array}{l}s_{\lambda}=0, s_{u} \neq 0 \\
s_{\lambda \lambda}-2 p_{\lambda} r_{\lambda} \neq 0\end{array}$ & $s_{u}$ & $-s_{\lambda \lambda}+2 p_{\lambda} r_{\lambda}$ & $(0, x)$ \\
\hline$n_{3}$ & $\begin{array}{l}s_{u}=0, s_{\lambda} \neq 0 \\
s_{u u}-2 p_{u} r_{u} \neq 0\end{array}$ & $s_{u u}-2 p_{u} r_{u}$ & $-s_{\lambda}$ & $(0, u x)$ \\
\hline$n_{4}$ & $\begin{array}{l}s_{u}=s_{\lambda}=0 \\
\rho_{1}=s_{u \lambda}-2\left(p_{\lambda} r_{u}-p_{u} r \lambda\right) \neq 0 \\
\rho_{2}=s_{\lambda \lambda}-2 p_{\lambda} r_{\lambda} \neq 0 \\
\rho_{3}=s_{u u}-2 p_{u} r_{u} \neq 0 \\
\rho_{1}^{2}-4 \rho_{2} \rho_{3} \neq 0 \\
\mu=\rho_{1} / 2\left(\rho_{2} \rho_{3}\right)^{1 / 2} \text { (modal) }\end{array}$ & $\rho_{3}$ & $\rho_{2}$ & $\begin{array}{l}(0, x) \\
(0, u x) \\
(0, u \lambda x)\end{array}$ \\
\hline
\end{tabular}

Next prove that

$$
\mathscr{S}=\left[\mathscr{M}^{2}+\langle v\rangle, \mathscr{M}, \mathscr{M}^{2}+\langle v\rangle, \mathscr{M}^{3}+\langle v\rangle\right] \subseteq \mathrm{RT}\left(n_{4}, \mathscr{U}\right)
$$

by verifying that $\mathscr{S} \subseteq \mathrm{RT}\left(n_{4}, \mathscr{U}\right)$ modulo $\mathscr{M} \mathscr{S}$ and then using Nakayama's Lemma. This inclusion is checked with a Maple program that computes determinants of all maximum-order minors of a non-square matrix. Maple is also used to prove that $\mathscr{S}$ is intrinsic. Hence, $\mathscr{S} \subseteq \mathscr{P}\left(n_{4}\right)$.

Step 2. Change coordinates modulo $\mathscr{S}$. The simplified expression, obtained by Maple, is given by $n_{4}$ in Table 1 .

Step 3. Find $\operatorname{cod} n_{4}$. Generators of $\mathrm{T}\left(n_{4}\right)$ modulo $\mathscr{S}$ are

$$
[0,1,0,0],[u, 0,0,0],[v, 0,0,0],[\lambda, 0,0,0],[0,0,0, v] \text {, }
$$

$[0,0, u, 0],[0,0, v, 0],[0,0, \lambda, 0],\left[0,0,0, \varepsilon_{1} u^{2}-\varepsilon_{2} \lambda^{2}+\mu u \lambda\right]$,

$$
\left[0,0,0,5 \varepsilon_{1} u^{2}-\varepsilon_{2} \lambda^{2}+\mu u \lambda\right], \quad\left[0,0,0,2 \varepsilon_{2} \lambda+\mu u\right], \quad[0,0,1,0], \quad[1,0,0,0] .
$$

It is easy to see that $[0,0,0,1],[0,0,0, u]$ and $[0,0,0, u \lambda]$ span the complement to $T\left(n_{4}\right)$ in $\overrightarrow{\mathscr{E}}_{\mathbf{O}(2)}\left(\mathrm{Fix} \mathbf{Z}_{2}\right)$. Therefore $\operatorname{cod} n_{4}=3$ and the topological codimension is 2 .

We end this subsection with bifurcation diagrams for the three least degenerate bifurcations. As usual, we plot $\lambda$ in the horizontal direction. Each diagram consists of a trivial solution branch $(x=0, y=0, \lambda)$ and another non-trivial solution branch $f(u, 0, \lambda)=0$. As in Remark 4.8, this is what we would have expected if we had performed a Liapunov-Schmidt reduction onto the kernel. We 
choose $\varepsilon_{1}=\varepsilon_{2}=1$ to draw the pictures. In Figure 2(a), we find the diagram for $n_{1}$, with a change in the eigenvalues as in Figure 1. Figure 2(b) depicts the diagram for the unfolding of $n_{2}$ given by

$$
\left(y,\left(u-\lambda^{2}+\alpha\right) x\right),
$$

when $\alpha>0$. The diagrams for $\alpha<0$ are obtained from those where $\alpha>0$ by changing $\varepsilon_{1} \rightarrow-\varepsilon_{1}$ and $\varepsilon_{2} \rightarrow-\varepsilon_{2}$. Figure 2(c) represents the diagram for the unfolding of $n_{3}$ given by

$$
\left(y,\left(u^{2}-\lambda+\alpha u\right) x\right),
$$

when $\alpha<0$. It is easy to see that when $\alpha>0$ the picture for the unfolding of $n_{3}$ is similar to diagram (a), but now with a quartic non-trivial solution branch.

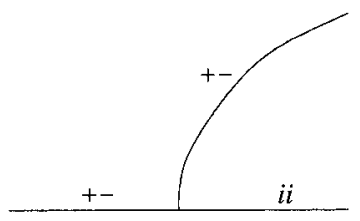

(a)

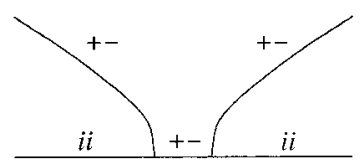

(b)

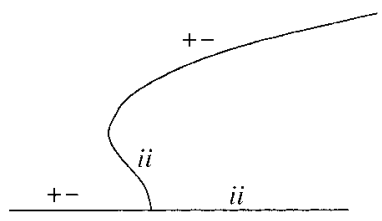

(c)

Figure 2. Bifurcation diagrams for (a) $n_{1}$, (b) the unfolding of $n_{2}$, and (c) the unfolding of $n_{3}$, when $\varepsilon_{1}=\varepsilon_{2}=1$.

\section{References}

1. R. Abraham, J. E. Marsden and T. Ratiu, Manifolds, tensor analysis and applications (Addison-Wesley, Reading, MA, 1983).

2. D. Armbruster and G. Dangelmayr, 'Coupled stationary bifurcations in non-flux boundary value problems', Math. Proc. Cambridge Philos. Soc. 101 (1987) 167-192.

3. V. I. ARNoLD, Ordinary differential equations (MIT Press, Cambridge, MA, 1973).

4. J. W. Bruce, A. A. Du Plessis and C. T. C. Wall, 'Determinacy and unipotency', Invent. Math. 88 (1987) 521-554.

5. D. Crawford, M. Golubitsky, M. G. M. Gomes, E. Knobloch and I. Stewart, 'Boundary conditions as symmetry constraints', Singularity theory and its applications, Warwick 1989 (ed. R. M. Roberts and I. N. Stewart), Vol. 2, Lecture Notes in Mathematics 1463 (Springer, Heidelberg, 1991) 63-79.

6. J. Damon, 'The unfolding and determinacy theorems for subgroups of $\mathscr{A}$ and $\mathscr{K}$ ', Mem. Amer. Math. Soc. 306 (1984).

7. G. Dangelmayr and D. Armbruster, 'Steady states mode interactions in the presence of $\mathbf{O}(2)$-symmetry and in non-flux boundary conditions', Multiparameter bifurcation theory (ed. M. Golubitsky and J. Guckenheimer), Contemporary Mathematics 56 (American Mathematical Society, Providence, RI, 1986) 53-68.

8. M. Field, M. Golubitsky and I. Stewart, 'Bifurcations on hemispheres', J. Nonlinear Sci. 1 (1990) 201-223.

9. H. FujiI, M. Mimura and Y. Nishiura, 'A picture of the global bifurcation diagram in ecological interacting and diffusing systems', Physica D 5 (1982) 1-42.

10. T. GAFFNEY, 'New methods in the classification theory of bifurcation problems', Multiparameter bifurcation theory (ed. M. Golubitsky and J. Guckenheimer), Contemporary Mathematics 56 (American Mathematical Society, Providence, RI, 1986) 97-116.

11. M. Golubitsky, J. E. Marsden and D. Schaeffer, 'Bifurcation problems with hidden symmetries', Partial differential equations and dynamical systems (ed. W. E. Fitzgibbon), Research Notes in Mathematics 101 (Pitman, San Francisco, 1984) 181-210.

12. M. GolubitSKy and D. SCHAEFFER, Singularities and groups in bifurcation theory, Vol. 1, Applied Mathematical Sciences 51 (Springer, New York, 1985).

13. M. Golubitsky, I. Stewart and D. SchaefFer, Singularities and groups in bifurcation theory, Vol. 2, Applied Mathematical Sciences 69 (Springer, New York, 1988).

14. M. G. M. Gomes and I. STEWART, 'Steady PDEs on generalized rectangles: a change of genericity in mode interactions', Nonlinearity 7 (1994) 253-272. 
15. G. W. Hunt, 'An algorithm for the nonlinear analysis of compound bifurcation', Philos. Trans. Roy. Soc. London 300 (1981) 443-471.

16. G. W. Hunt, 'Symmetries of elastic buckling', Engrg. Struct. 4 (1982) 21-28.

17. M. Manoel and I. Stewart, 'Degenerate bifurcations with $\mathbf{Z}_{2} \oplus \mathbf{Z}_{2}$-symmetry', Internat. J. Bifur. Chaos Appl. Sci. Engrg. to appear.

18. J. N. Mather, 'Stability of $\mathbf{C}^{\infty}$ mappings, III. Finitely determined map germs', Inst. Hautes Études Sci. Publ. Math. 35 (1968) 127-156.

19. I. Melbourne, 'The recognition problem for equivariant singularities', Nonlinearity 1 (1988) 215-240.

20. A. L. Onishchick and E. B. Vinberg, Lie groups and algebraic groups (Springer, Heidelberg, 1990).

21. V. Pò̀naru, Singularités $C^{\infty}$ en présence de symétrie, Lecture Notes in Mathematics 510 (Springer, Berlin, 1986).

22. G. Schwarz, 'Smooth functions invariant under the action of a Lie group', Topology 14 (1975) 63-68.

Míriam Manoel

ICMC-USP

Caixa Postal 668

São Carlos

13.560-970

Brazil

miriam@icmc.sc.usp.br
Ian Stewart

Mathematics Institute

University of Warwick

Coventry CV4 7AL

ins@maths.warwick.ac.uk 